\title{
Experimental verification of optical backhaul links for high- altitude platform networks: Atmospheric turbulence and downlink availability
}

\author{
Joachim Horwath*,†, Nicolas Perlot, Markus Knapek and Florian Moll \\ German Aerospace Centre (DLR), Institute of Communications and Navigation, Weßling, Germany
}

\begin{abstract}
SUMMARY
Optical backhaul downlinks from high-altitude platforms (HAPs) are investigated. An experiment demonstrated the advantages of optical links: a small and lightweight terminal with low power consumption was launched to the stratosphere and data transmitted down to a ground station at a rate of $1.25 \mathrm{Gbit} / \mathrm{s}$. Owing to the chosen system parameters and the high budget margin, disturbing turbulence effects did not decrease the link performance.

The scientific aspect of the experiment was to study turbulence effects in order to design future systems with higher transmission performance. On the day of the experiment, measured scintillation and wavefront distortions were minimal in the morning. The best atmospheric conditions were observed about $3 \mathrm{~h}$ after sunrise with a peak of the atmospheric coherence length $r_{0}$ at $16 \mathrm{~cm}$. An $r_{0}$ of $4 \mathrm{~cm}$ was measured as the worst case before sunrise and later during the day. This trend could also be observed for power- $\left(\sigma_{p}^{2}\right)$ and intensity scintillation index $\left(\sigma_{I}^{2}\right)$. The latter $\sigma_{I}^{2}$ changed from 0.28 (best case) to 1.12 . For small $\sigma_{I}^{2}$, a lognormal intensity probability density function was measured.

Apart from the robust intensity modulation scheme with direct detection which was used for the trial, future improved systems could benefit from a coherent transmission scheme. According to the $r_{0}$ measurements and further simulations on heterodyne efficiency it turned out that the aperture size can be decreased from 40 to $10 \mathrm{~cm}$ without any significant change in the link margin.

Future stratospheric optical links between HAPs or links from platforms to satellites will not suffer from cloud blockage but it remains an issue for up/downlinks to a ground station. This can be mitigated by ground-station diversity. Four optical ground stations in the southern part of Europe can lead to an availability of over $98 \%$. The separation distance of the ground stations is about $900 \mathrm{~km}$ with a negligible correlation of cloud cover. A change of wavelength from the employed 1.55 to a wavelength around $11-\mu \mathrm{m}$ with minimum cloud attenuation would increase the link availability for thin clouds. Copyright (C) 2007 John Wiley \& Sons, Ltd.
\end{abstract}

Accepted 23 June 2007

KEY WORDS: $\quad$ stratospheric optical payload experiment; turbulence effects; optical downlink availability

\footnotetext{
*Correspondence to: Joachim Horwath, German Aerospace Centre, Institute of Communication and Navigation, P.O. Box 11 16, D-82230 Weßling, Germany.

†E-mail: joachim.horwath@dlr.de

Contract/grant sponsor: European Commission; contract/grant number: FP6-IST-2003-506745
}

Copyright (C) 2007 John Wiley \& Sons, Ltd. 


\section{INTRODUCTION}

High-altitude platforms (HAPs) that hover in a quasi-geostationary manner at an altitude of $18-25 \mathrm{~km}$ combine advantages of satellite and terrestrial communications infrastructure. This includes short deployment time, easy equipment upgrade, flexible capacity increase, ability of substantial indoor coverage, and a geographical coverage of hundreds of kilometers. The European CAPANINA project is developing communications technologies for use with aerial platforms with the aim of delivering 'broadband for all.' The emphasis is on hardto-reach users and those disadvantaged by geography [1]. Investigated services like fixed broadband wireless access up to $120 \mathrm{Mbit} / \mathrm{s}$ to end users require a broadband backhaul network [2]. Free-space optical (FSO) communication technology has a major potential to complement microwave technology for the backhaul traffic. High data rates in the range of Gbit/s are the highest motivation to use FSO systems. However, low terminal size and weight, small aperture sizes and low power consumption are clear advantages for HAP systems. High attenuation due to clouds is not a limiting factor for inter-platform and platform-to-satellite links, the main application of HAP-FSO. Due to a maximum cloud ceiling of $13 \mathrm{~km}$ for mid-latitude locations, inter-platform link distances of up to $900 \mathrm{~km}$ are possible with $100 \%$ availability [3].

In order to design reliable optical terminals, stratospheric tests are necessary. The stratospheric optical payload experiment (STROPEX), a part of CAPANINA, was one step in this direction by gaining system design and operations experience and by gathering atmospheric index-of-refraction turbulence data. The experiment was focused on experimental verification of the chosen acquisition, pointing, and tracking systems, measurement of atmospheric impacts and successful verification of a broadband downlink from a stratospheric testbed (balloon/aerodynamic HAP aircraft). Although the optical link between HAP and ground station is affected by clouds, it is gaining interest in operational scenarios. Apart from the higher data rate and lower size and weight, optical terminals consume less power which is a limited resource on HAPs. The optical up/downlink could then work in diversity with a microwave link in a stand-alone HAP scenario. In a multiple-HAP network with inter-platform or platform-to-satellite links, cloud cover diversity will be used to cope with cloud blockage.

In Section 2, the technical aspects of STROPEX are introduced and the developed hardware including the stratospheric optical terminal and the optical ground station is explained. The operations and main achievements of the trial, namely the Gbit transmission from the stratosphere and the system tracking performance are highlighted in Section 3. The investigation of atmospheric turbulence, that is the scientific aspect of the experiment, is considered in Section 4. The principle of operation of the two instruments to analyze the received field is explained and an approach to derive turbulence profiles for this novel scenario is introduced. The measurement results of wave front distortion, scintillation, intensity probability density functions (PDFs), and turbulence profiles are discussed.

The link budget and a possible improvement based on gathered technical trial expertise are explained in Section 5. A comparison with the performance of a different modulation scheme is conducted. The important question of availability due to clouds for these optical up/ downlink scenarios is answered in Section 6. Also, the wavelength-dependent attenuation for two different common cloud types is discussed for the scenario. The paper concludes with Section 7. 


\section{THE STRATOSPHERIC OPTICAL PAYLOAD EXPERIMENT}

\subsection{System concept}

The overall system concept for STROPEX was to mount a free-space experimental laser terminal (FELT) on a stratospheric balloon that would fly to an altitude of approximately $22 \mathrm{~km}$. A downlink would then take place between the FELT and a transportable optical ground station (TOGS) as depicted in Figure 1.

On the basis of various factors such as component availability and atmospheric attenuation [4], three different wavelengths were chosen for the lasers. The TOGS used beacon lasers at $810 \mathrm{~nm}$ to illuminate the FELT. The FELT had two beacon lasers around $977 \mathrm{~nm}$ and a communication laser at $1550 \mathrm{~nm}$. At $1550 \mathrm{~nm}$, the atmospheric absorption is negligible at clear air conditions [4].

Intensity modulation (IM, on/off-keying) with direct detection (DD), common in terrestrial fiber-optical communication, was the chosen transmission scheme. An IM/DD scheme is advantageous because there is a range of available components with proven reliability, e.g. laser diodes, fiber amplifiers, detectors and receiver electronics. According to theory, 10 incoming photons per bit (mean) are sufficient for an uncoded bit error rate (BER) of $10^{-9}$ [5]. However, in practical systems using standard APD (avalanche photo diode) detectors, the receiver

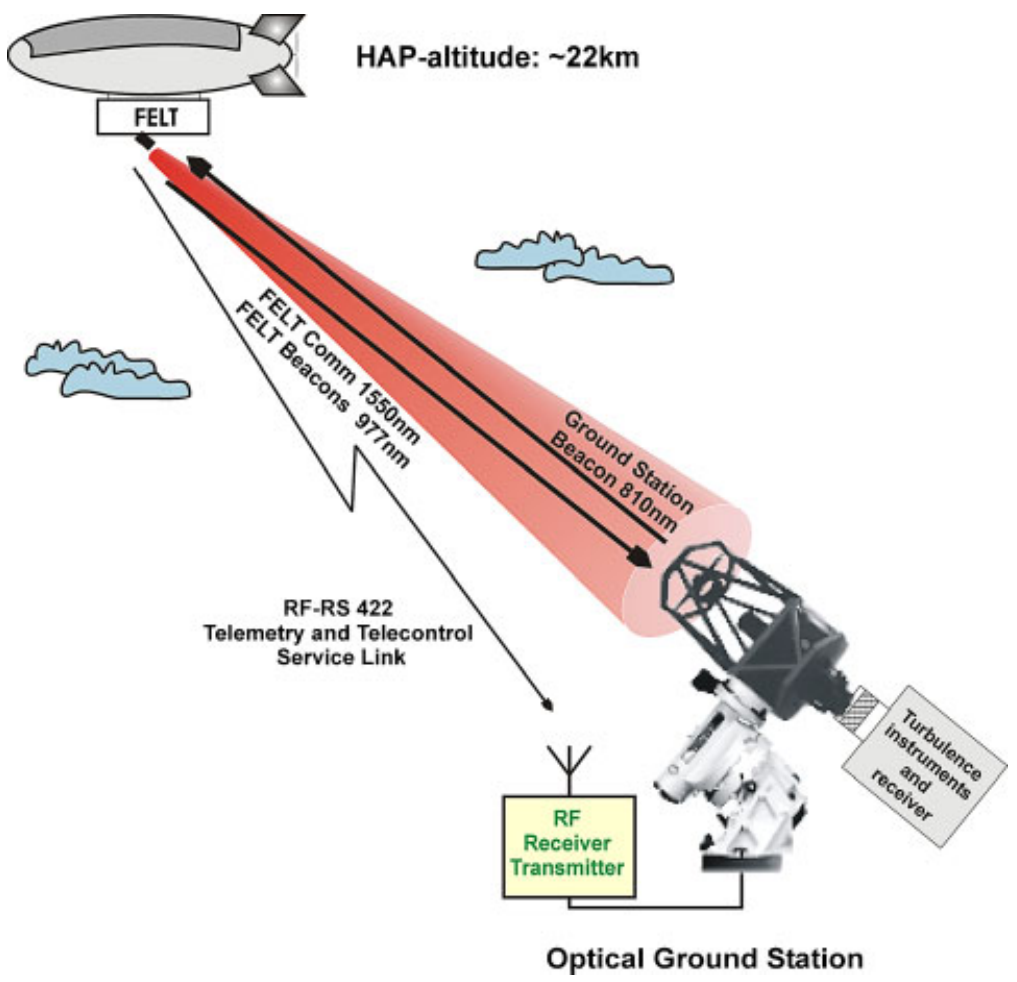

Figure 1. STROPEX scenario. 
sensitivity is usually not better than several 100 photons per bit due to thermal receiver noise and other degrading electronic effects. The actual sensitivity of the frontend developed for STROPEX was measured at 168 photons per bit at a data transmission rate of $1.25 \mathrm{Gbit} / \mathrm{s}$ and a BER of $3 \times 10^{-7}$. This is due to the less than optimal sensitivity of the APD, thermal noise and degrading electronic effects.

\subsection{Free-space optical terminal (FELT)}

The FELT was an optical transmission terminal developed mainly for the CAPANINA stratospheric experiments. The following were the primary design constraints for the FELT:

- Stratospheric environmental conditions (temperatures down to $-70^{\circ} \mathrm{C}$ and near vacuum conditions).

- High stratospheric carrier rotation speeds (nine rotations per minute).

- Lightweight and streamlined design.

- Autonomous and robust acquisition capability in the presence of strong background light.

The final FELT design is shown in Figure 2 with a side access panel removed. The structural subsystem consisted of an aluminum base plate which was also used for passive thermal control. The final terminal weight was $17.54 \mathrm{~kg}$. The housing consisted of a carbon fiber sandwich structure that reduced the weight and maximized thermal insulation properties. Additionally, the housing was designed to easily interface with an aerodynamic HAP (i.e. a stratospheric airplane). The outer dimension of the terminal was $78 \times 33 \mathrm{~cm}$.

Figure 3 shows the bottom side of the mounting plate, where the electrical power subsystem along with the beacon laser diodes and their supporting electronics (diode drivers and thermal control) are mounted. For redundancy, the FELT used two independent co-aligned beacon systems at 977.53 and $977.8 \mathrm{~nm}$, each with $200 \mathrm{~mW}$ output power. One of the beacons was coupled with the $1550 \mathrm{~nm}$ signal light and used the same transmission optics. The pointing assembly consisted of a two-axis optical periscope along with the supporting encoders, DC motors and motor drivers. The pointing resolution was $8.7 \mu \mathrm{rad}$ with a maximum rotation speed of $240^{\circ} / \mathrm{s}$ for the main axis and $120^{\circ}$ s for the elevation axis. The acceleration was better than

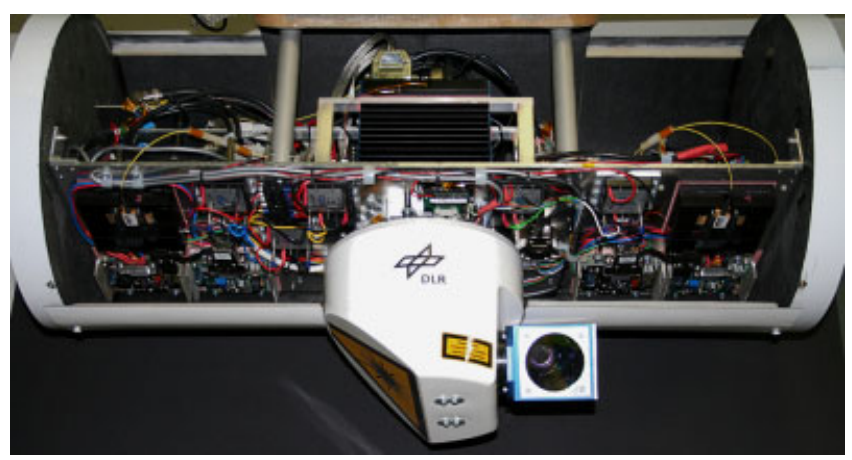

Figure 2. Assembly photo of FELT with opened housing. 


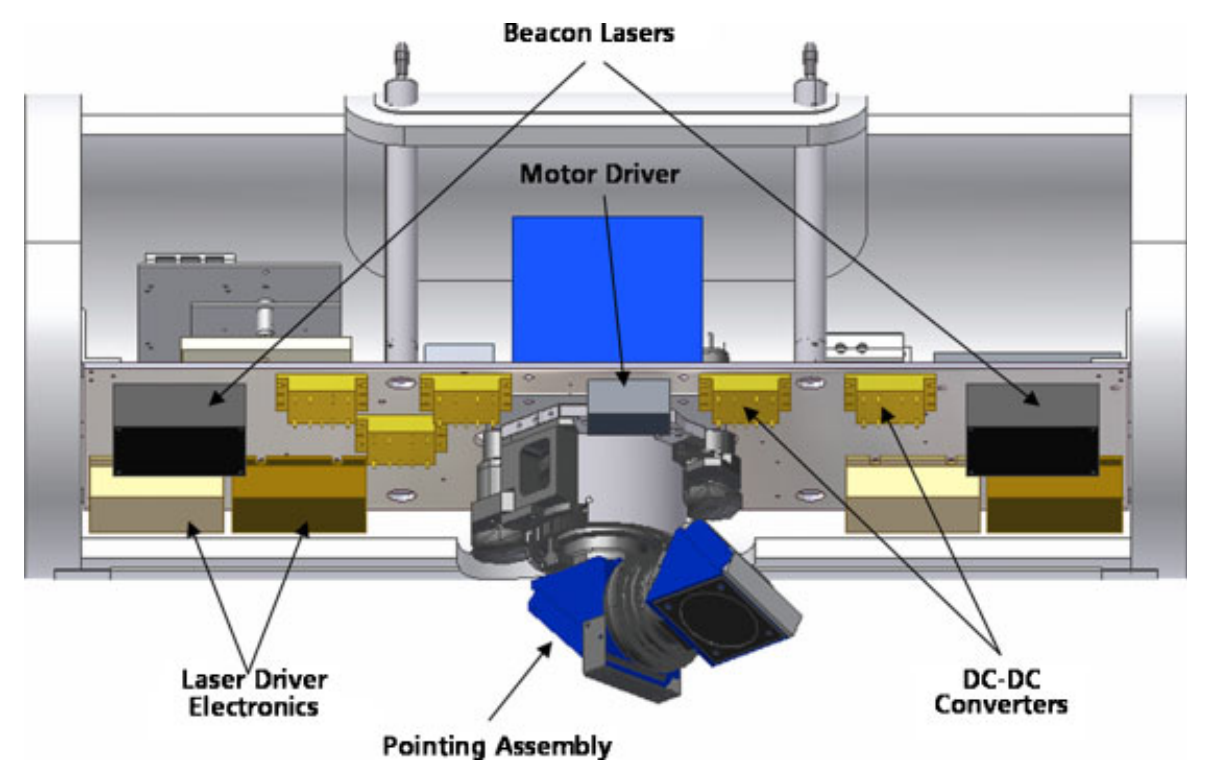

Figure 3. CAD sketch of FELT-bottom side.

$250^{\circ} / \mathrm{s}^{2}$. The design driver for the dynamic properties of the pointing system was the possible high spin rate of the stratospheric carrier. Five DC-DC converters of the electronic power system were also mounted on the bottom side of the plate.

The components mounted on the top of the plate can be seen in Figure 4. An onboard telemetry and tele-command (TMTC) subsystem enabled control of the terminal via RS422 connection and included the monitoring of 16 temperature probes. The signal electronics generated a pseudo random binary sequence (PRBS) with a sequence length of $10^{23}-1$ and an adjustable data rate of up to $1.25 \mathrm{Gbit} / \mathrm{s}$. This data source drove a laser diode module with $1 \mathrm{~mW}$ output power which was optically amplified to a transmission power of $100 \mathrm{~mW}$. The beam was routed into the pointing assembly via the optical assembly, which consisted of the laser collimators, optical components and the fast CMOS tracking camera with a $4^{\circ}$ field of view (FOV) [6]. The pointing assembly was controlled by the FELT pointing acquisition and tracking (PAT) computer. The PAT computer took inputs from on-board gyroscopes, the tracking camera and the periscope encoders, and controlled the motion of the pointing assembly.

\subsection{Transportable optical ground station (TOGS)}

The FELT was designed to transmit data to the TOGS, which is shown in Figure 5. The central component of the ground station was a $40 \mathrm{~cm}$ Cassegrain telescope. The optical communications receiver and the atmospheric turbulence measurement devices are mounted on an optical bench attached to the telescope [7].

Figure 6 shows a sketch of the optical ground station receive path. The beam from the $40 \mathrm{~cm}$ Cassegrain telescope is collimated and split up by beam splitter cubes (BSC) to the measurement 


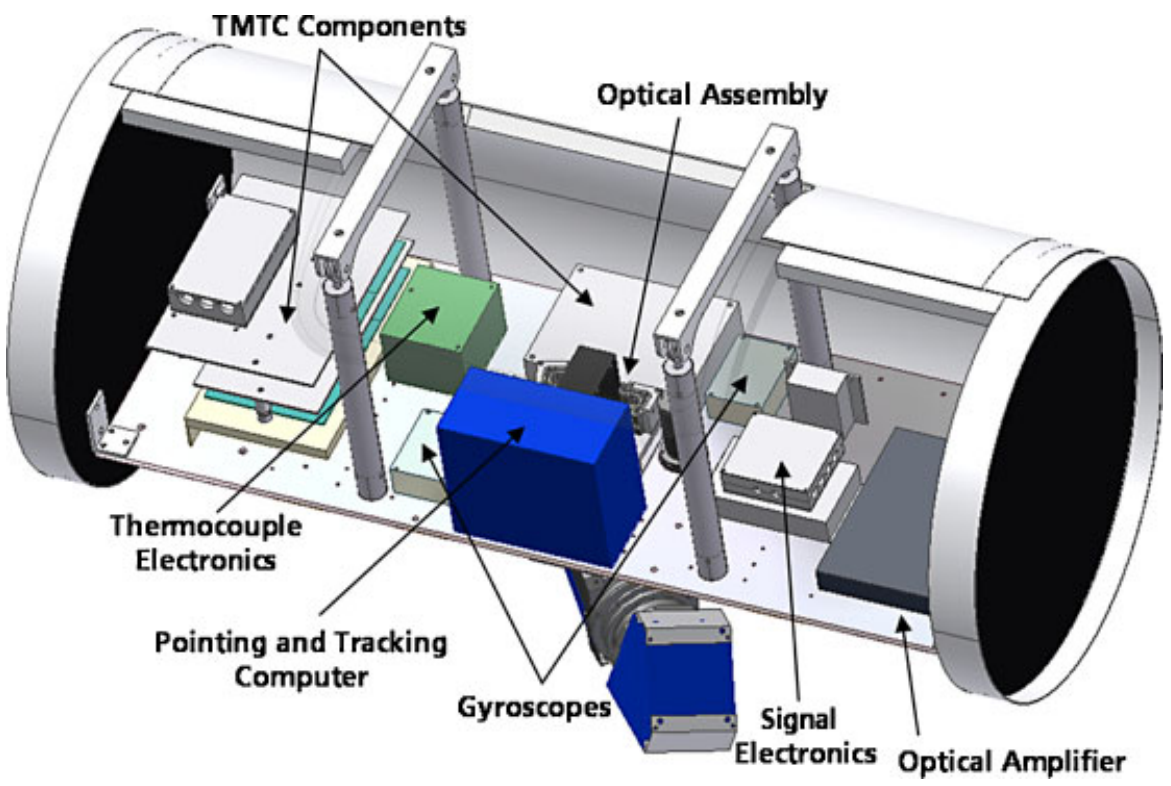

Figure 4. CAD sketch of FELT-top side.

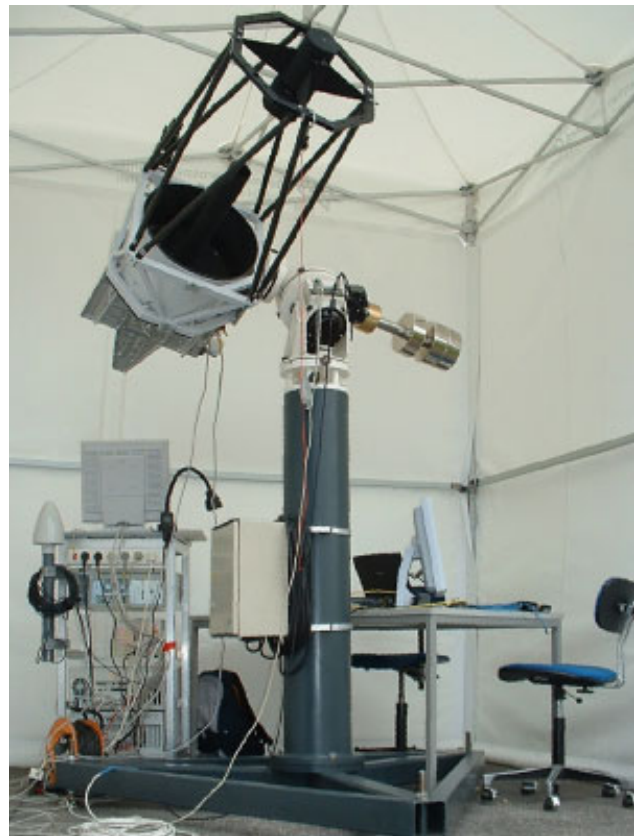

Figure 5. Transportable optical ground station (TOGS). 


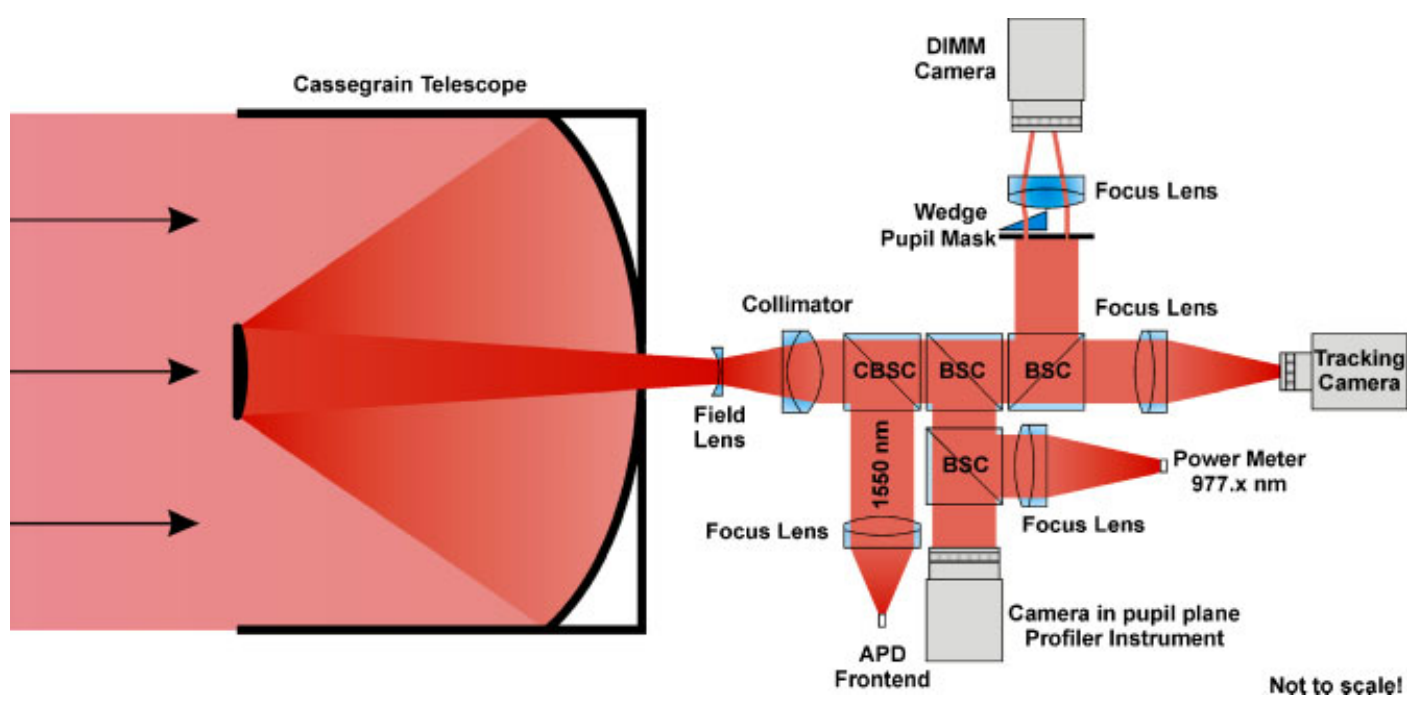

Figure 6. Details of the optical bench with the optical path for tracking camera and measurement instruments. Measurement instruments: differential image motion monitor (DIMM) and turbulence profiler instrument.

instruments and the camera of the tracking system. The first BSC of the optical system is a chromatic one (CBSC). This CBSC deflects the $1550 \mathrm{~nm}$ beam to the communication receiver with the APD communication frontend. All other instruments, the differential image motion monitors (DIMM) camera, the profiler instrument, the tracking camera and power meter worked with the $977 \mathrm{~nm}$ beacon light emitted by the stratospheric terminal. This approach had two main advantages. The received $1550 \mathrm{~nm}$ light could be used for the communication receiver only. All other instruments could use silicon technology. The variety of available cameras especially with high sensitivity and short exposure time is higher for this technology and wavelength range. The turbulence instruments (turbulence profiler, TP, and DIMM) are explained in Section 4.

\section{TRIAL OPERATIONS AND ACHIEVEMENTS}

The Launch and operations took place at the ESRANGE facilities in northern Sweden near Kiruna. A $12000 \mathrm{~m}^{3}$ piloted stratospheric balloon carrying the FELT was launched at 3:54 a.m. on 30.08.2005 and flew for approximately $8.5 \mathrm{~h}$ before parachuting to the ground. During the mission acquisition, data transmission tests and atmospheric measurements were carried out. Figure 7 shows a picture of the experiment before take off. The nacelle which housed the FELT was located at the bottom of a $70 \mathrm{~m}$ flight train freely suspended on the launch vehicle during launch. Figure 8 shows the separation distance between the TOGS and the FELT. The maximum range during the mission was $64.15 \mathrm{~km}$ and occurred at 07:03 a.m. local time. The altitude was about $23 \mathrm{~km}$. 


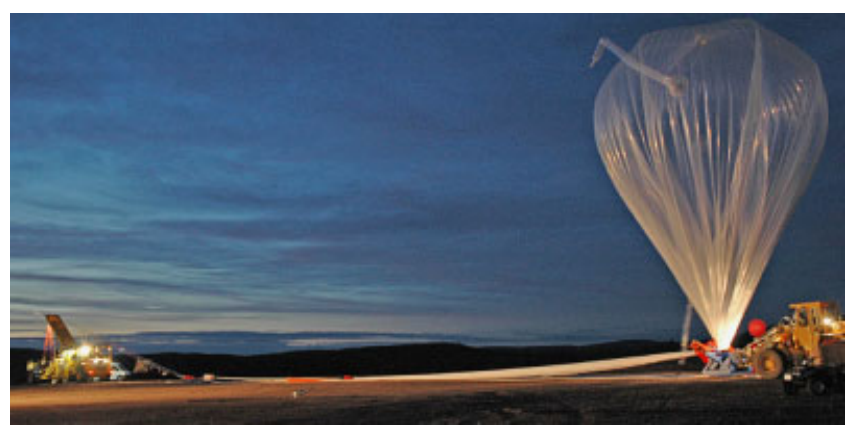

Figure 7. The experiment before launch.

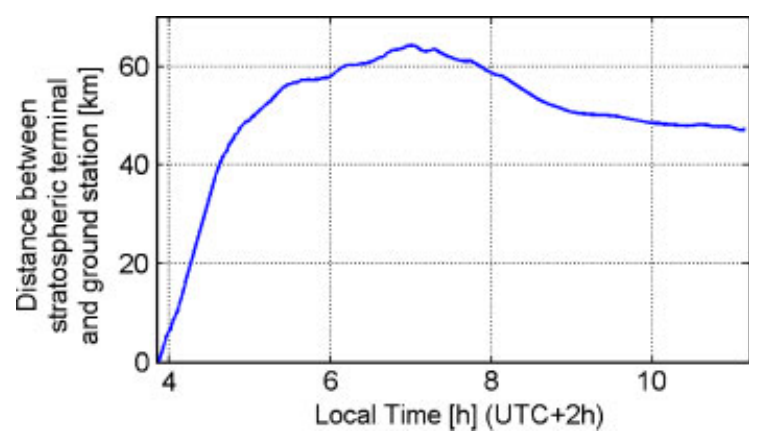

Figure 8. Distance between the optical ground station and the stratospheric terminal during the trial.

\subsection{Acquisition and Tracking}

FELT acquisition of the ground station worked very reliably during night and day conditions. The acquisition time depended on the pointing assembly scan speed, but even with a slow scan speed of $10^{\circ} \mathrm{s}$, the maximal acquisition time was below $30 \mathrm{~s}$. Figure 10 shows the stratospheric system after acquisition. The FELT exhibited very precise tracking capability in stratospheric conditions. Figure 9 shows a cumulative density function of the FELT tracking deviation over the $8 \mathrm{~h}$ of data collection during the trial. Seventy-five percent of the time, the FELT tracking deviation was less than $142 \mu \mathrm{rad}\left(0.0081^{\circ}\right)$ which corresponded to 1 pixel on the FELT tracking camera. During the other $25 \%$ of the time, mostly acquisition tests with different beacon powers or FELT parameter setups were performed, or the FELT could not track the ground station due to stratospheric wind shear that caused a large pendulous motion of the nacelle. Compared to the high beam divergence of $1 \mathrm{mrad}$, the margin of the tracking system was very high and did not lead to a remarkable decrease of communication system performance.

\subsection{Data transmission results}

The FELT was capable of data transmission at three different rates: $270 \mathrm{Mbit} / \mathrm{s}, 622 \mathrm{Mbit} / \mathrm{s}$ and $1.25 \mathrm{Gbit} / \mathrm{s}$. BERs were measured for the 270 and $622 \mathrm{Mbit} / \mathrm{s}$ links; however, due to the 


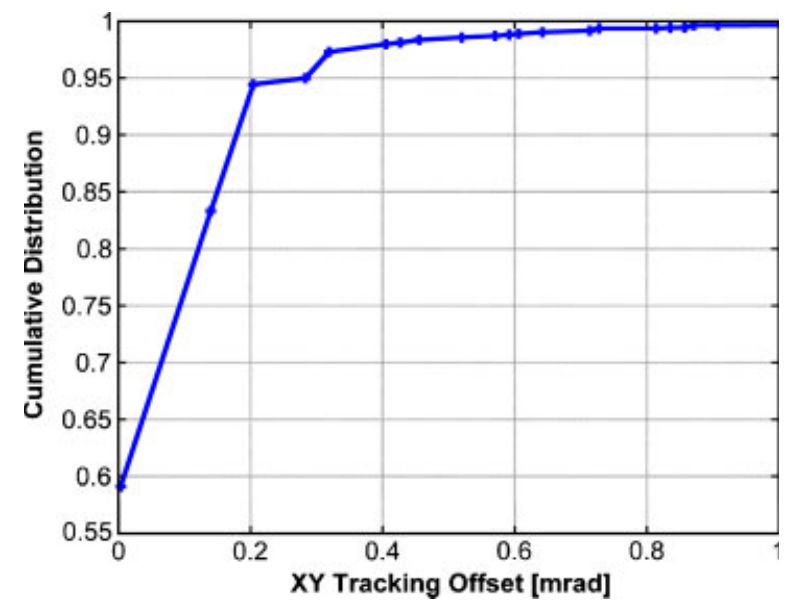

Figure 9. Cumulative density function of FELT tracking deviation.

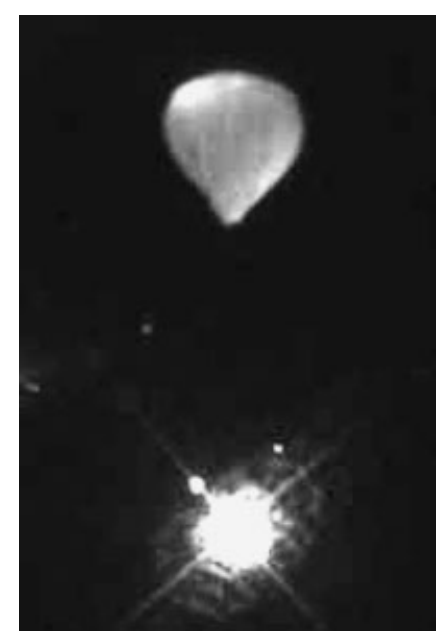

Figure 10. Near infrared picture of the stratospheric carrier with FELT from ground-station tracking camera after acquisition at approximately $60 \mathrm{~km}$ range.

limited bandwidth of the BER test receiver, no direct BER measurements could be performed at $1.25 \mathrm{Gbit} / \mathrm{s}$.

At $622 \mathrm{Mbit} / \mathrm{s}$, the high signal-to-noise ratio enabled a BER of less than $10^{-9}$ within an unlimited measurement interval. Frequently, the BER went down to $10^{-10}$ for several minutes. This high quality signal could be received up to a maximum distance of $64.15 \mathrm{~km}$ from the ground station. The $622 \mathrm{Mbit} / \mathrm{s}$ eye pattern can be seen in Figure 11. The data transmission at $1.25 \mathrm{Gbit} / \mathrm{s}$ was also successful. The eye pattern of the received $1.25 \mathrm{Gbit} / \mathrm{s}$ signal is shown in Figure 12. 


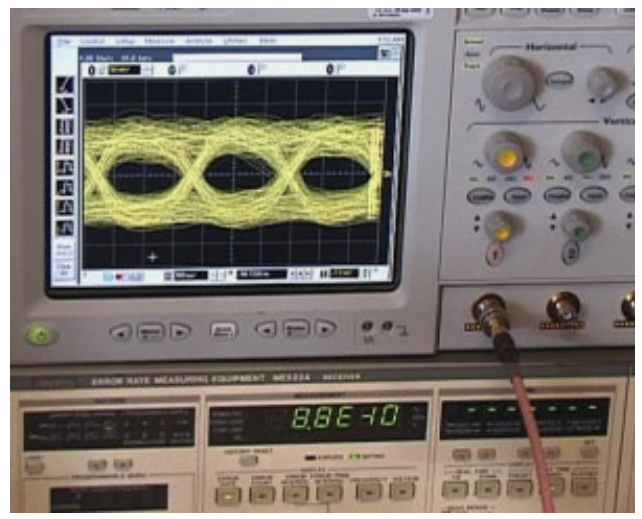

Figure 11. Received PRBS signal eye pattern at $622 \mathrm{Mbit} / \mathrm{s}$ with bit error rate measurement receiver.

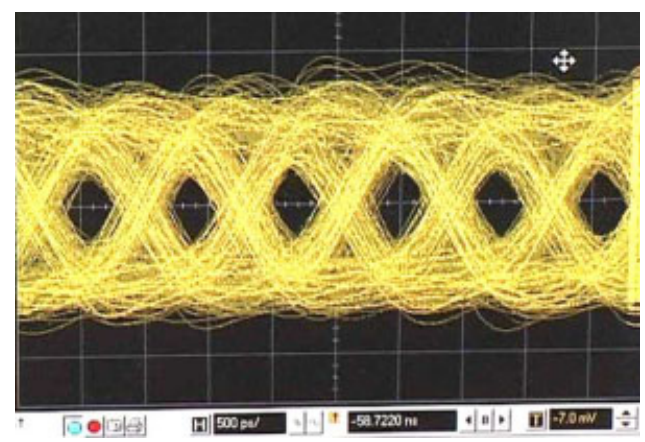

Figure 12. Received PRBS signal eye pattern at $1.25 \mathrm{Gbit} / \mathrm{s}$.

During the transmission tests, several thick clouds disturbed testing from time to time. Several very thick black clouds completely prevented communication. Thin white clouds did not severely affect the transmission, but did lead to a slight closing of the eye pattern and an increased BER.

Late in the morning, most of the clouds disappeared resulting in near clear sky conditions. During that time, the angular separation between the sun and the balloon was very small at about $5^{\circ}$ and blue sky. Despite an increase in background radiation which could have affected the signal reception, no impairment of link quality was observed. This positive effect was achieved by the narrow filtering $(10 \mathrm{~nm})$ of the incoming $1550 \mathrm{~nm}$ signal and the reduced sun radiance at this near-infrared (NIR) wavelength. This highlights a clear advantage of the $1550 \mathrm{~nm}$ technology over common NIR wavelengths like $850 \mathrm{~nm}$.

\section{ATMOSPHERIC TURBULENCE INSTRUMENTS AND TRIAL MEASUREMENT RESULTS}

One goal of the trial was to evaluate the impact of the index-of-refraction turbulence. Turbulent air volumes have different temperatures and thus different indices of refraction. These air 
volumes along the propagation path act like thin lenses which focus and defocus the beam. The wave intensity at the receiving telescope forms a speckle pattern (Figure 13, left). This speckle pattern or spatial intensity redistribution changes in time due to the wind and turbulent mixing process. The turbulence-induced distortions of the optical field result in a fading channel that depends on the modulation type. Optical turbulence is quantified by means of the refractiveindex structure constant $C_{n}^{2}$ which scales the statistical variations of the refractive index $n . C_{n}^{2}$ is related to the structure function $D_{n}$ of the refractive index by [8]

$$
D_{n}(\rho)=C_{n}^{2} \rho^{2 / 3}
$$

where $\rho$ is the separation distance between two points in the air.

The size and the strength of the speckles have a significant impact on an incoherent (e.g. IM/ DD) transmission scheme. The resulting fades and surges can be reduced by increasing the receiving aperture and thereby averaging the spatial intensity fluctuations.

The phase distortions (which are not visible in Figure 13, left) are characterized by the transverse coherence length and can greatly deteriorate the performance of coherent receivers (e.g. BPSK). This is particularly the case when the transverse coherence length is smaller than the receiver aperture (see Section 5.2).

Two instruments were integrated in the optical ground station to sense the received optical field. The TP estimates the statistics of the received intensity and derives from them the $C_{n}^{2}$ profile along the propagation path. The DIMM measures the image dancing of two focused spots (Figure 13, right) and estimates the wavefront distortion which is represented by the atmospheric coherence length $r_{0}$.

\subsection{Turbulence profiler instrument}

The TP is based on the single-star profiler [9-11]. The single-star profiler relies on the correspondence between the $C_{n}^{2}$ turbulence profile on the transmission path and the intensity spatial covariance function in the aperture of the receiver. Distinct turbulence layers along the path have different influences on the intensity covariance function $B(\rho)$ of the received field. Therefore, the $C_{n}^{2}$ profile can be reconstructed from the covariance function. However, this correspondence between covariance function and $C_{n}^{2}$ profile relies on an ill-conditioned relation, as the altitude of the $C_{n}^{2}$ has a rather weak influence on the covariance-function shape. Therefore, profiles can be restored with only a limited resolution of the propagation path.
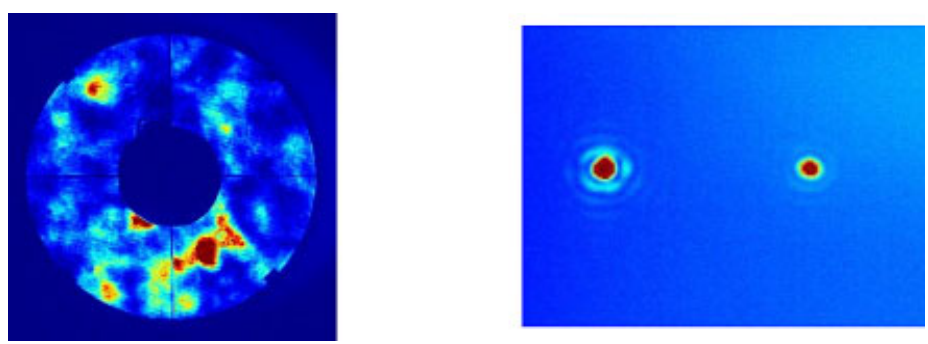

Figure 13. Example of gathered turbulence raw data. Recorded intensity snapshot of the $40 \mathrm{~cm}$ Cassegrain telescope aperture with central obscuration in the turbulence profiler pupil (left). Recorded DIMM sample picture with the two focal spots (right). 
The covariance function $B(\rho)$ of the separation distance $\rho$ in the receiver aperture is related to the profile $C_{n}^{2}(z)$ under weak fluctuations by

$$
B(\rho)=\int_{0}^{L} C_{n}^{2}(z) W(z, \rho) \mathrm{d} z
$$

where $W(z, \rho)$ is the wave-dependent kernel of the transformation. In the experiment, the transmitted beam was nearly a spherical wave and the transformation kernel has been assumed to be equal to [12].

$$
W(z, \rho)=0.033 \cdot 8 \pi^{2} k^{2} \int_{0}^{\infty} \kappa^{-8 / 3} J_{0}\left(\kappa \rho \cdot\left(1-\frac{z}{L}\right)\right)\left(1-\cos \left[\frac{\kappa^{2} z}{k}\left(1-\frac{z}{L}\right)\right]\right) \mathrm{d} \kappa
$$

where $k=2 \pi / \lambda$ is the wave number, $z$ the distance from the receiver, $L$ the link range, and $J_{0}$ denotes the Bessel function of first kind and zero order. Figure 14 shows the kernel $W(z, \rho)$ according to Equation (2) for the downlink beacon of the STROPEX experiment $(\lambda=977 \mathrm{~nm}$ and $L=60 \mathrm{~km}$ ). This function was used for the reconstruction of the turbulence profile. The kernel decreases to zero for both ends of the optical link, which implies that the instrument becomes insensitive to turbulence close to the transmitter and the receiver.

It should also be pointed out that the measured spatial covariance function $B(\rho)$ measured by the TP contains the scintillation index given by $\sigma_{I}^{2}=B(0)$ and the mean speckle size $\rho_{c}$ usually defined by $B\left(\rho_{c}\right) / B(0)=e^{-1}$.

\subsection{Differential image motion monitor}

The DIMM derives the $r_{0}$ parameter from the estimated variance of the differential motion of two spots $[13,14]$. These two spots are produced by letting the received light go through two separate apertures in the entrance pupil of the telescope. The light from the two apertures is

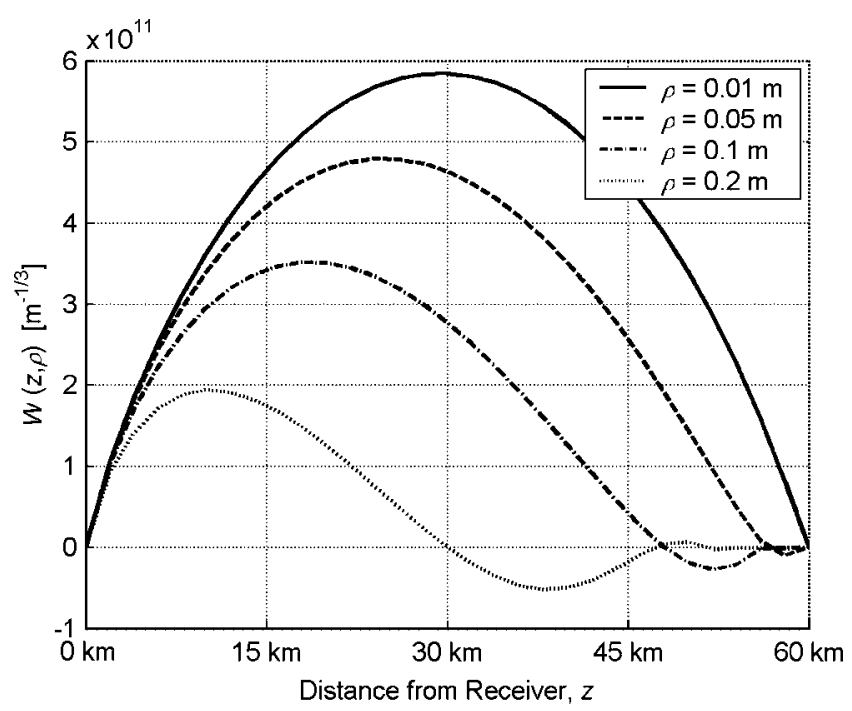

Figure 14. Transformation kernel of the turbulence profiler for $\lambda=977 \mathrm{~nm}$ and $L=60 \mathrm{~km}$. 
focussed by a lens, and one of the paths is transmitted through an optical wedge (Figure 6) to obtain two distinct spots on a charge-coupled device (CCD) camera (as shown in Figure 13, right). This instrument is mainly used by astronomers to measure $r_{0}$ at observatory sites [15].

An advantage of the $r_{0}$ estimation based on DIMM measurements is that this technique is inherently insensitive to tracking errors. This characteristic is especially important for a dynamic scenario like STROPEX.

\subsection{Results from the measurement instruments}

The measurements during the balloon trial started at 6:13 a.m. (local time) and ended at 11:01 a.m. During this period, 35 synchronous measurements of DIMM and profiler were performed.

The estimation of the $r_{0}$ parameter from the DIMM over the daytime is shown in the upper graph of Figure 15. An $r_{0}$ peak of up to $16 \mathrm{~cm}$ with the best atmospheric conditions can be seen at about 8:00 a.m. The second curve in Figure 15 shows the $r_{0}$ values reconstructed from the $C_{n}^{2}$
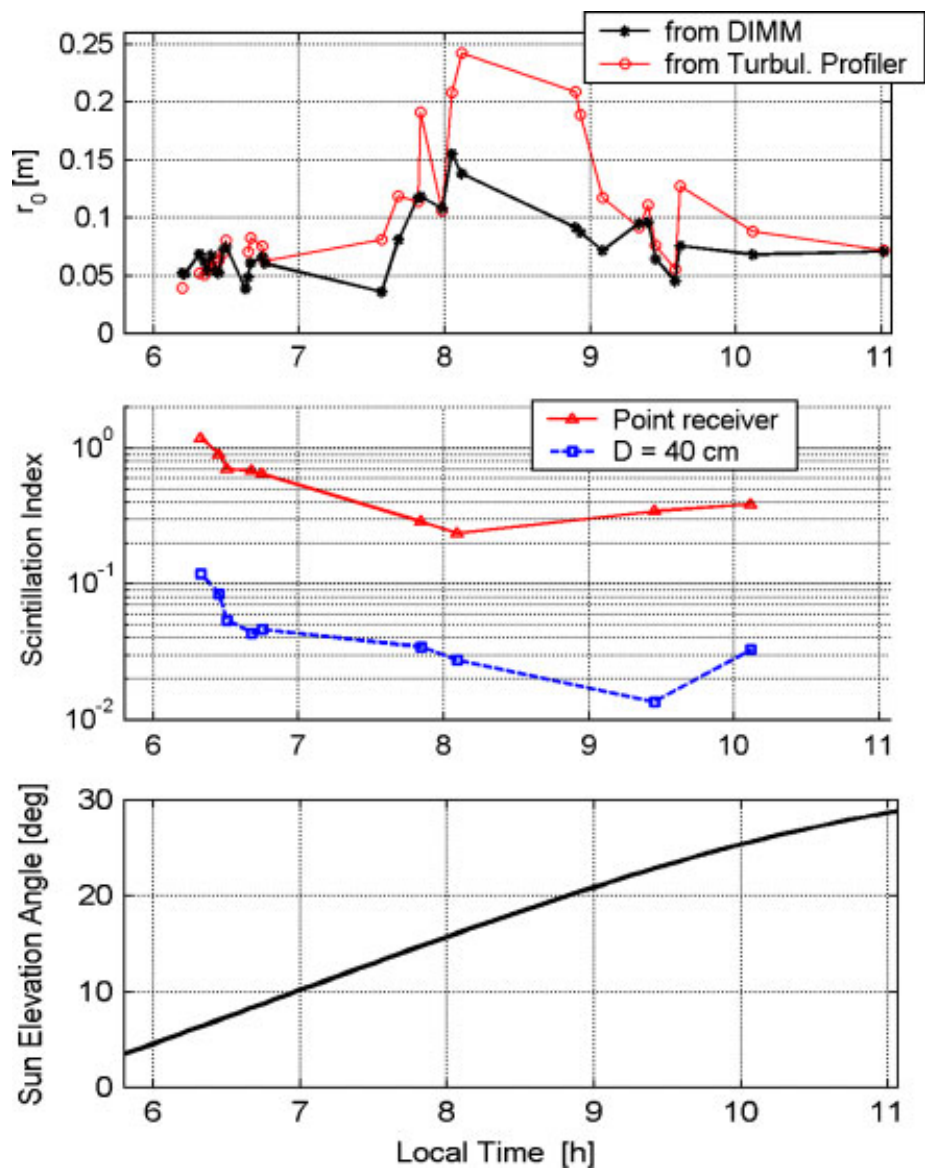

Figure 15. Atmospheric coherence length $r_{0}$ (upper graph), scintillation index (middle graph) and sun elevation angle (lower graph) for the main trial period between 6:00 and 11:00 a.m. local time. 
profiles measured by the TP. These reconstructed values confirm the development of $r_{0}$ and also show the increase between 8 and 9 a.m.; however, the reconstruction from the TP most of the time overestimates $r_{0}$.

The atmospheric coherence length $r_{0}$ is reconstructed from the $C_{n}^{2}$ values with a spherical wave by [12]

$$
r_{0}=2.1 \cdot\left[1.46 \cdot k^{2} \cdot \int_{0}^{L} C_{n}^{2}(z) \cdot(1-z / L)^{5 / 3} \mathrm{~d} z\right]^{-3 / 5}
$$

where $z$ is the distance from the receiver. As given by Equation (4), $r_{0}$ is mainly influenced by turbulence close to the receiver. However, the TP cannot estimate the $C_{n}^{2}$ values close to the receiver. Therefore, the $r_{0}$ as derived by the reconstructed profile might become overestimated due to underestimated $C_{n}^{2}$ values near the receiver, where they have the strongest impact on $r_{0}$. This might explain the difference of the $r_{0}$ values of DIMM and TP shown in Figure 15.

The middle graph in Figure 15 shows the scintillation index defined as the variance of the irradiance normalized to its mean. We show the scintillation index for two aperture sizes: a point aperture and the $40-\mathrm{cm}$ telescope aperture. The scintillation index over a point aperture is referred to as the intensity scintillation index and is noted by $\sigma_{I}^{2}$, whereas that over the $40-\mathrm{cm}$ aperture is referred to as the power scintillation index and is noted by $\sigma_{P}^{2}$. The intensity scintillation index was evaluated from the pixel samples of the profiler camera. Both the intensity scintillation index and the power scintillation index show a minimum around 8:00. This corroborates the larger $r_{0}$ values and thus the decrease of atmospheric turbulence during this hour. The turbulence minimum occurs approximately $2 \mathrm{~h}$ after sunrise.

The lower graph of Figure 15 shows the sun elevation angle for the experiment day 30th August 2005. Sunrise was at 5:15 a.m. local time. The drop in the turbulence level occurs when the air and the ground have the same temperature and less turbulence is created by energy transfer processes.

Figure 16 shows the PDF of the normalized intensity, measured at about 8:03 and corresponding to the weakest intensity fluctuations measured during the experiment. The intensity scintillation index was $\sigma_{I}^{2}=0.28$ and the lognormal distribution featuring this variance fits the measured distribution relatively well; this fit is in agreement with the weak-fluctuation theory valid for $\sigma_{I}^{2}<1[12]$.

The intensity PDF for the strongest measured fluctuations can be seen in Figure 17. The scintillation index was $\sigma_{I}^{2}=1.12$. Fits with two different theoretical distribution models are shown in the graph. As expected, the lognormal fit is not as good as in Figure 16. Under strong fluctuations the gamma-gamma distribution, which stems from the product of two independent gamma variables with mean of unity, has been proposed [16]. This distribution features two parameters, $\alpha$ and $\beta$. However, even by considering the most suitable distribution parameters $(\alpha=\beta=2.2)$, the gamma-gamma distribution does not provide a good fit.

Figure 18 is a three-dimensional plot of seven selected turbulence profiles measured during the trial. The characteristic decrease of turbulence strength with increasing altitude and distance from receiver, respectively, can be seen for all profiles. This tendency can be best seen for the profile measured at 7:34 o'clock local time, where the distance between the ground station and the stratospheric terminal was close to the maximum transmission distance of the trial. 


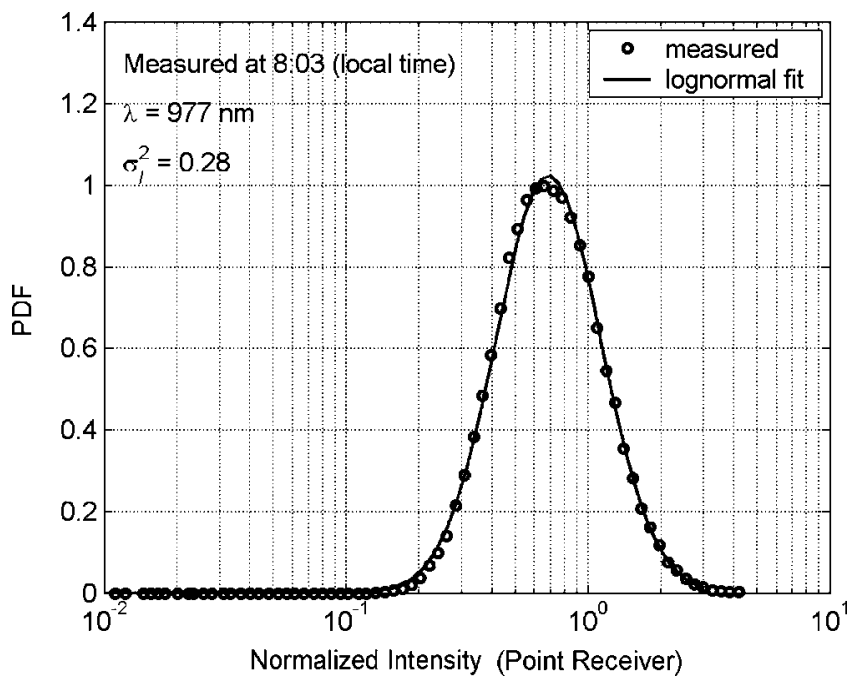

Figure 16. Intensity PDF measured at 8:03 o'clock local time for the moment of the weakest turbulence during the trial and comparison with a lognormal fit.

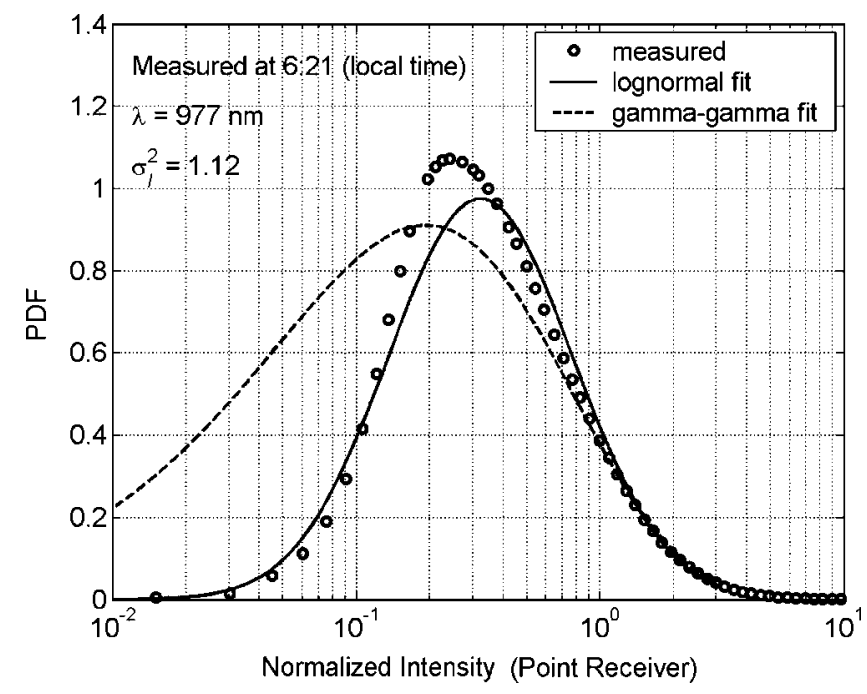

Figure 17. Intensity PDF measured at 6:21 o'clock local time for the moment of the strongest turbulence during the trial and comparison with two different fits.

\section{LINK BUDGET AND POSSIBLE IMPROVEMENTS ON FUTURE HAP-TO-GROUND LINKS}

\subsection{Budget elements for atmospheric optical link}

Before presenting the link budget of the chosen scenarios, we briefly review the different budget elements for FSO links as these may differ from the classical RF elements. 


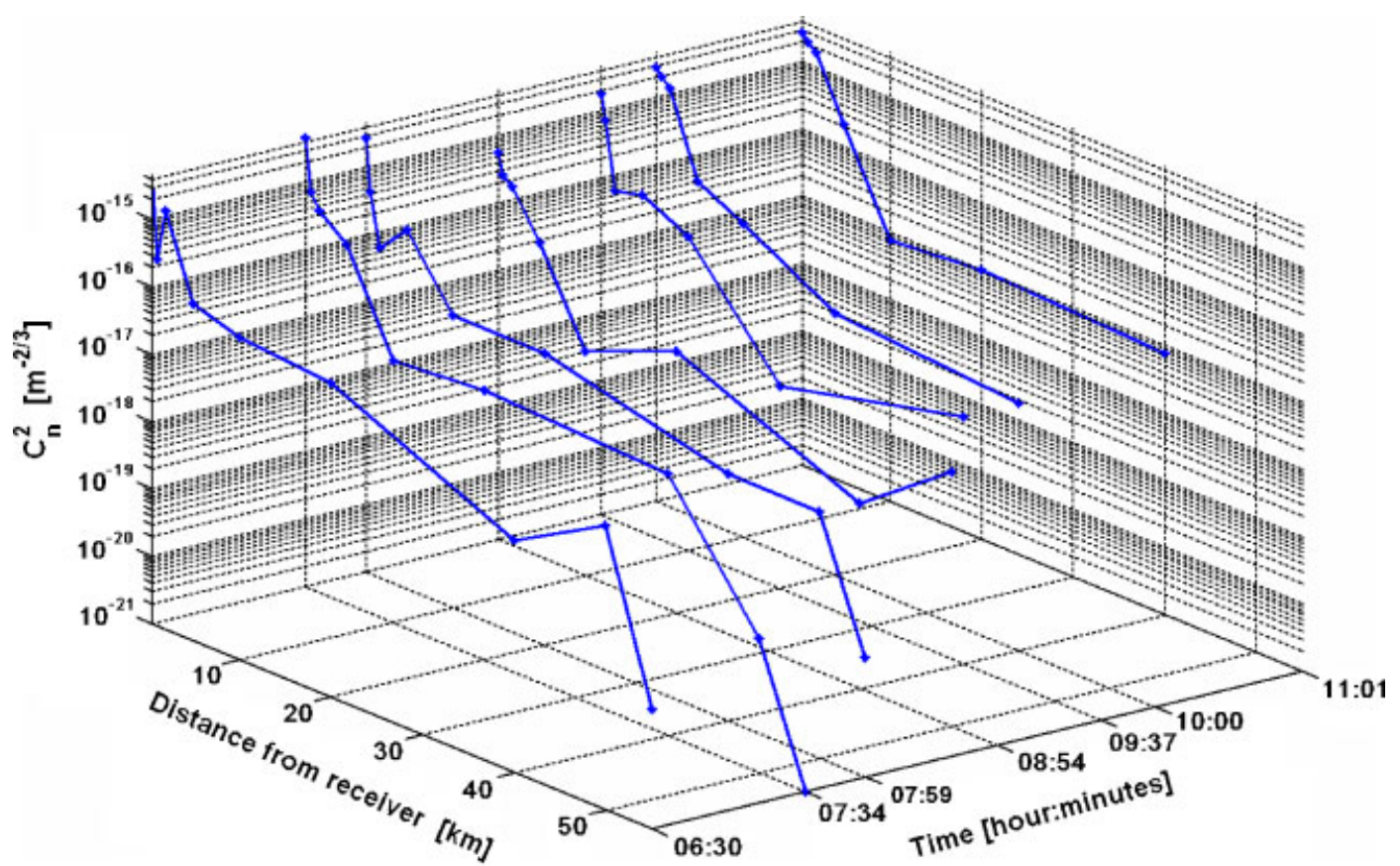

Figure 18. Three-dimensional plot of seven selected $C_{n}^{2}$ turbulence profiles measured during the trial between 6:13 and 11:01 a.m. local time. The profiles were estimated from 30-s measurements with the TP, which equals about 180 frames.

5.1.1. Basic link elements. In Figure 19, the basic geometrical considerations are sketched. Tx and $\mathrm{Rx}$ denote the transmitter and receiver, respectively. $L$ is the transmission distance and $\theta$ is the full divergence angle of the transmitted beam. $D$ is the receiver aperture diameter.

With $\Theta$ the solid angle associated with $\theta$, the effective transmitter gain $G_{\mathrm{Tx}}$ is given by [17]

$$
G_{\mathrm{Tx}}=\frac{4 \pi}{\Theta} \approx \frac{16}{\theta^{2}} \quad \text { with } \theta \ll 1
$$

We define the receiver gain as the effective angular extent of the receiver. With $\alpha$ as the angle over which the receiver is viewed by the transmitter and $\Gamma$ the corresponding solid angle, the receiver gain is given by

$$
\begin{aligned}
G_{\mathrm{Rx}} & =\frac{\Gamma}{4 \pi} \approx \frac{\alpha^{2}}{16} \quad \text { with } \alpha \ll 1 \\
& \approx\left(\frac{D}{4 L}\right)^{2}
\end{aligned}
$$

Note that according to some other definitions, Equation (6) may combine the receiver gain and the free-space loss [17]. With $P_{\mathrm{Tx}}$ the transmitted power, the received power $P_{\mathrm{Rx}}$ of a basic optical link is thus given by $P_{\mathrm{Rx}}=P_{\mathrm{Tx}} G_{\mathrm{Tx}} G_{\mathrm{Rx}}$. 


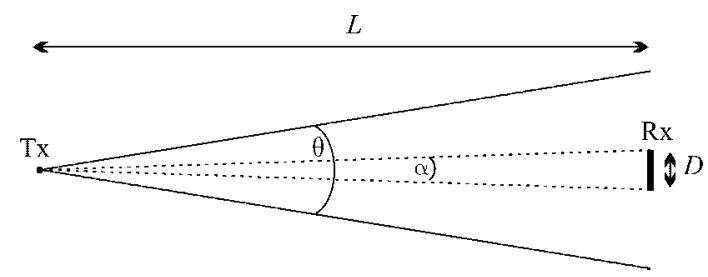

Figure 19. Basic geometrical considerations for transmission and detection of an optical beam.

5.1.2. Background light. For FSO links the sun is the most powerful source of background radiation and measures must be taken to decrease its impact. The background-light penalty or loss depends on the level of the received background light with respect to the received signal level. It thus increases with the FOV of the receiver. Along the same lines as in [18], loss caused by a blue background sky was evaluated at $-2.5 \mathrm{~dB}$ for a field of view of $100 \mu \mathrm{rad}$ and an optical-filter bandwidth of $10 \mathrm{~nm}$.

5.1.3. Pointing loss. With $\theta$ being the full beam divergence and $\sigma_{\text {point }}$ being the root mean square (RMS) pointing deviation angle over a single axis, the pointing loss is mainly determined by the ratio $\theta / \sigma_{\text {point }}$. It can be shown that when the beam has a Gaussian profile and when the pointing jitter angle follows a two-dimensional Gaussian distribution, the fluctuating power follows a beta distribution [19]. The CDF of the normalized power $P$ is then

$$
F_{P, \text { point }}(P)=P^{\left(\theta / 4 \sigma_{\text {point }}\right)^{2}}, \quad 0 \leqslant P \leqslant 1
$$

In our link budget, we define the pointing loss as the value of $P$ giving $F_{P \text {,point }}(P)=0.01$.

5.1.4. Scintillation loss. To be able to evaluate the scintillation loss for the communication beam, we must estimate the power scintillation index $\sigma_{P, 1550 \mathrm{~nm}}^{2}$ of spherical wave with a wavelength of $1550 \mathrm{~nm} . \sigma_{P, 1550 \mathrm{~nm}}^{2}$ can be derived from the scintillation parameters measured at $\lambda=977 \mathrm{~nm}$ using the scintillation theory. The Rytov theory predicts that the intensity scintillation indices $\sigma_{I, \lambda_{1}}^{2}$ and $\sigma_{I, \lambda_{2}}^{2}$ at $\lambda_{1}$ and $\lambda_{2}$, respectively, are related by [12]

$$
\sigma_{I, \lambda_{2}}^{2}=\sigma_{I, \lambda_{1}}^{2}\left(\frac{\lambda_{2}}{\lambda_{1}}\right)^{-7 / 6}
$$

yielding

$$
\sigma_{I, 1550 \mathrm{~nm}}^{2}=0.58 \sigma_{I, 977 \mathrm{~nm}}^{2}
$$

It is also known that the mean speckle size increases with the square root of the wavelength:

$$
\rho_{c, \lambda_{2}}=\rho_{c, \lambda_{1}}\left(\frac{\lambda_{2}}{\lambda_{1}}\right)^{1 / 2}
$$

yielding

$$
\rho_{c, 1550 \mathrm{~nm}}=1.26 \rho_{c, 977 \mathrm{~nm}}
$$


When the receiving aperture is much larger than the correlation lengths $\rho_{c}$, the power scintillation index of $\lambda_{1}$ is related to that of $\lambda_{2}$ by

$$
\sigma_{P, \lambda_{2}}^{2}=\sigma_{P, \lambda_{1}}^{2} \frac{\sigma_{P, \lambda_{2}}^{2}}{\sigma_{P, \lambda_{1}}^{2}}\left(\frac{\rho_{c, \lambda_{1}}}{\rho_{c, \lambda_{2}}}\right)^{2} \text { with } D \gg \rho_{c}
$$

which reduces to

$$
\sigma_{P, \lambda_{2}}^{2} \approx \sigma_{P, \lambda_{1}}^{2} \text { with } D \gg \rho_{c}
$$

with the approximation $\left(\lambda_{2} / \lambda_{1}\right)^{1 / 6} \approx 1$. In STROPEX, the intensity scintillation index of the beacon beam $(\lambda=977 \mathrm{~nm})$ varied between 1.1 and 0.25 , and the power scintillation index varied between 0.12 and 0.013 (see Figure 15). The estimated mean speckle size was between 3 and $5 \mathrm{~cm}$ for the beacon wavelength of $977 \mathrm{~nm}$. Based on these estimations, we will hold Equation (13) a valid.

In our budget calculation, we assume a lognormal distribution of the scintillating power and, similar to the pointing loss, we define the scintillation loss as the fade level under which the signal remains for $1 \%$ of the time [20].

5.1.5. Heterodyne efficiency. When a coherent detection is performed, the signal suffers from turbulence-induced wavefront distortions which degrade the so-called heterodyne efficiency $\eta_{\text {het }}$ $[21,22]$. The heterodyne efficiency is comprised between 0 and 1 and measures the field match of the local oscillator with the received field at a given time and is mostly determined by phase fluctuations. Because the signal-to-noise ratio is proportional to $\eta_{\text {het }} P$, with $P$ the received optical power, the heterodyne efficiency can be viewed as a loss in the link budget. It has been shown that correlation between phase and amplitude decreases rapidly as the distance of propagation through turbulence increases [8]. For a long propagation path of $60 \mathrm{~km}$, we ignore this correlation and we separate the turbulence loss into the heterodyne-efficiency loss and the scintillation loss. To find the loss associated with the heterodyne efficiency, we must first find the PDF of the heterodyne efficiency. For that, we resorted to simulations in which samples of the phase field $S(\boldsymbol{\rho})$, where $\boldsymbol{\rho}$ is a two-dimensional vector, have been generated [23]. These generated phase fields are Gaussian and feature the well-known $r_{0}$-dependent structure function $D_{S}(\rho)$ [8]:

$$
D_{S}(\rho)=6.88\left(\frac{\rho}{r_{0}}\right)^{5 / 3}
$$

The generated fields with distorted wavefronts have been applied to a coherent receiver with different aperture diameters $D$ [24]. To reproduce the behavior of a realistic coherent receiver, the angle of arrival of the wave on the aperture has been removed, yielding a tilt-removed phase front $S_{\text {tilt-rem }}(\boldsymbol{\rho})$. The heterodyne efficiency was then calculated as

$$
\eta_{\text {het }}=\left|\int_{\text {Aperture }} \exp \left(S_{\text {tilt-rem }}(\boldsymbol{\rho})\right) \mathrm{d} \boldsymbol{\rho}\right|^{2}
$$

The PDFs of the square root of $\eta_{\text {het }}$ for different aperture sizes $D$ can be seen in Figure 20. It can be seen from the PDFs that the fades remain acceptable for $D / r_{0}<2$. Similar to previous losses, we define the heterodyne-efficiency loss as the fade level under which the signal remains for $1 \%$ of the time. 


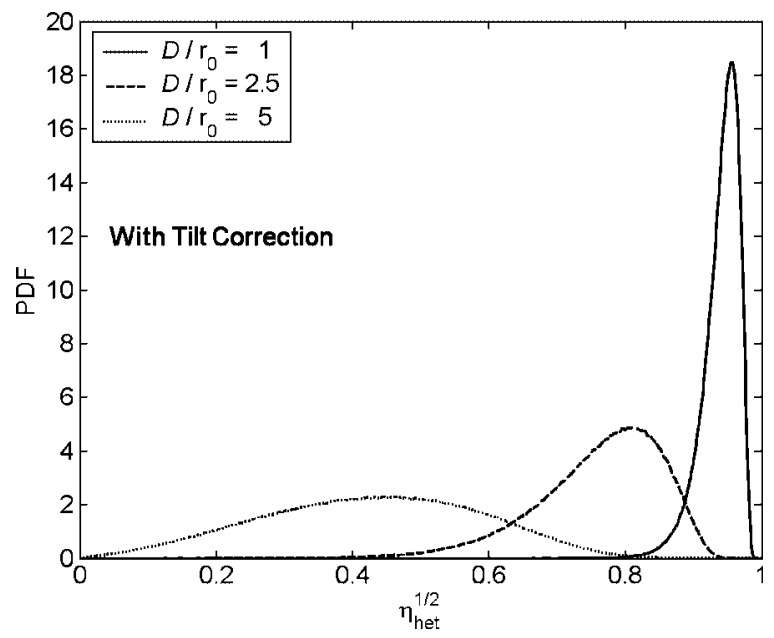

Figure 20. PDF of the square root of the heterodyne efficiency. The distributions were obtained from the simulation of distorted wavefronts. Different ratios of the receiver aperture $D$ to atmospheric coherence length $r_{0}$ are considered.

Table I. Link budget of the STROPEX communication downlink compared to a coherent link budget with decreased aperture size is shown.

\begin{tabular}{lcc}
\hline Budget elements & Capanina (Incoherent) & Coherent \\
\hline Tx optical power & 0.10 & $0.10 \mathrm{~W}$ \\
Tx divergence angle & $1.0 \mathrm{E}-03$ & $1.0 \mathrm{E}-03 \mathrm{rad}$ \\
Link distance $L$ & $6.0 \mathrm{E}+04$ & $6.0 \mathrm{E}+04 \mathrm{~m}$ \\
Rx-aperture diameter & $4.0 \mathrm{E}-01$ & $\mathbf{1 . 0 E}-\mathbf{0 1} \mathrm{m}$ \\
Tx optical power & 20 & $20 \mathrm{dBm}$ \\
Tx Gain & 72.0 & $72.0 \mathrm{dBi}$ \\
Rx Gain & -115.6 & $-\mathbf{1 2 7 . 6} \mathrm{dBi}$ \\
Pointing loss (Prob $=1 \mathrm{E}-2)$ & -1.6 & $-1.6 \mathrm{~dB}$ \\
Background-light loss & -2.5 & $\mathbf{0 . 0} \mathrm{dB}$ \\
Atmospheric attenuation & -2.6 & $-2.6 \mathrm{~dB}$ \\
Scintillation loss (Prob $=1 \mathrm{E}-2)$ & -3.3 & $\mathbf{- 5 . 8} \mathrm{dB}$ \\
Heterodyne efficiency (Prob $=1 \mathrm{E}-2)$ & & $-\mathbf{2 . 4} \mathrm{dB}$ \\
Rx optical loss & -3.0 & $-3.0 \mathrm{~dB}$ \\
(Equivalent) Received power & -36.5 & $-50.9 \mathrm{dBm}$ \\
Required Rx-power (BER $=1 \mathrm{E}-9)$ & $-\mathbf{4 4 . 9}$ & $-\mathbf{5 4 . 0} \mathbf{d B m}$ \\
Link margin & $\mathbf{8 . 4}$ & $\mathbf{3 . 0 ~ d B}$ \\
\hline
\end{tabular}

\subsection{STROPEX link budget and comparison with a coherent link}

The reviewed link budget of STROPEX is given in the second column of Table I. During the experiment, the 1550-nm communication beam had a divergence of $\theta=1 \mathrm{mrad}$ and the singleaxis RMS pointing error was $\sigma_{\text {point }}=70 \mu \mathrm{rad}$ (see also Section 3.1). For the scintillation loss, we consider the relatively pessimistic case of $\sigma_{P}^{2}=0.12$. STROPEX featured a link margin of about 
$8.4 \mathrm{~dB}$. Note that, in reality, the combination of beam jitter, scintillation and heterodyne efficiency leads to a compound distribution of the fluctuating signal, which may correspond to slightly different losses (see for example [25]). It is therefore an approximation to consider the different losses independently as we have done here.

An idea to improve future HAP feeder links would be to use an advanced transmission scheme like coherent BPSK. In terms of receiver sensitivity, coherent detection is unrivaled for high data rate links in space applications. In the shot-noise-limited case, the difference is small ( 9 photons/bit for coherent and 10 photons/bit for incoherent systems to achieve BER $=10^{-9}$ ). But practical coherent systems work closer to that of the theoretical limit. Background radiation can be neglected due to the extremely small-noise bandwidth of the receiver which is in the order of the data bandwidth (e.g. 1-GHz signal bandwidth corresponds to only about $0.0035 \mathrm{~nm}$ optical bandwidth for $1 \mu \mathrm{m}$ wavelength). Accordingly, data reception with the sun in the FOV would be possible. Note, however, that the STROPEX system, which featured incoherent detection, could dispose of the background radiation by means of a $10-\mathrm{nm}$ optical filter. This bandwidth was sufficiently small to track the stratospheric terminal as close as about $5^{\circ}$ close to the sun without remarkable decrease of BER (Section 3.1).

It has been demonstrated experimentally that coherent detection could also be used for long atmospheric links [26]. Considering the coherent detection for the HAP downlink scenario, we present the associated link budget in the third column of Table I. To keep the heterodyne efficiency reasonably high, the size of the receiving aperture must approximately match the $r_{0}$ parameter. To derive an $r_{0}$ value from our measurements, we note that parameters $r_{0, \lambda_{2}}$ and $r_{0, \lambda_{1}}$ at wavelengths $\lambda_{1}$ and $\lambda_{2}$, respectively, are related by [9]

$$
r_{0, \lambda_{2}}=r_{0, \lambda_{1}}\left(\frac{\lambda_{2}}{\lambda_{1}}\right)^{6 / 5}
$$

Therefore, the $r_{0}$ for $1550 \mathrm{~nm}$ is related to the $r_{0}$ measured at $977 \mathrm{~nm}$ by $r_{0,1550 \mathrm{~nm}}=1.74 r_{0,977 \mathrm{~nm}}$. Choosing the measured value $r_{0,977 \mathrm{~nm}}=4.0 \mathrm{~cm}$ (see Figure 15), we obtain the value $r_{0,1550 \mathrm{~nm}}=$ $7.0 \mathrm{~cm}$ for our link budget. Accordingly, the diameter of the receiving aperture was taken as $10 \mathrm{~cm}$, resulting in a heterodyne efficiency of $-2.4 \mathrm{~dB}$. With a smaller aperture, the scintillation loss for the coherent link is higher than for the incoherent link because less aperture averaging occurs [16]. A scintillation loss of -5.8 was estimated. Finally, to calculate the required optical power, we consider a rather realistic coherent receiver that requires, on average, 25 photons per bit to achieve a BER of $10^{-9}$ [27]. This coherent link, although feasible, has a lower link margin than the STROPEX link.

\section{AVAILABILITY OF HAP UP/DOWNLINKS}

\subsection{Cloud coverage in Europe}

In the atmosphere, clouds are the most disturbing factors regarding the attenuation of the light beam. Most of the clouds attenuate so strongly that there cannot be a link established when passing through. Therefore, it is beneficial to identify specific ground station locations with low probability of cloud occurrence. This section deals with cloud coverage statistics and link availabilities using data from the European Cloud Climatology (ECC) Project. These data were derived from the advanced very high resolution radiometer (AVHRR) sensors on board of the NOAA (National Oceanic and Atmospheric Administration) satellites. The sensors work with 


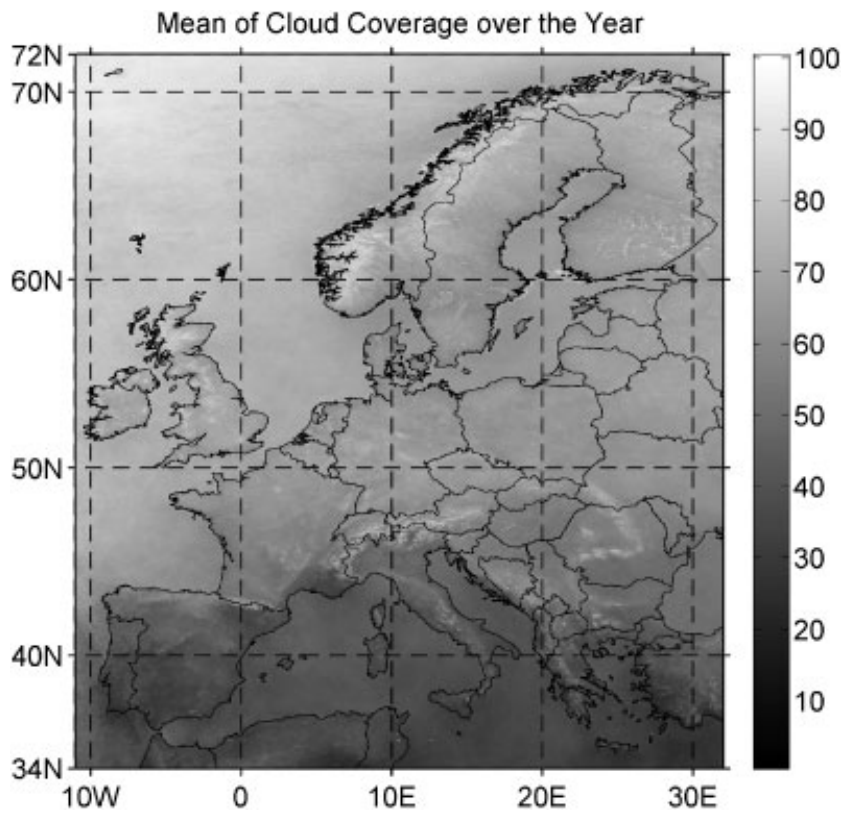

Figure 21. Average cloud coverage in Europe derived from ECC data. The values are a mean over the year.

a resolution of about $1.1 \times 1.1 \mathrm{~km}^{2}$ which is high enough for the purpose being addressed. Figure 21 shows the mean cloud coverage of a year in Europe based on data from the years $2000 / 04 / 05$. One can see in the figure the typical north-south decrease in mean cloud coverage. Hence, the amount of cloud occurrence in higher latitudes is bigger than in lower ones. For instance, Kiruna has a mean coverage of $74 \%$ and Calar Alto of $38 \%$. Due to this fact, one is supposed to prefer locations in southern Europe for setting up an optical ground station. In the case of a ground-station diversity concept this should count for all stations.

Because cloud coverage in Europe is for example higher in winter months than in summer months, we subdivide the year into its quarterly periods:

Quarter 1: January, February, March

Quarter 2: April, May, June

Quarter 3: July, August, September

Quarter 4: October, November, December

Figure 22 illustrates the mean cloud coverage corresponding to each quarter. For instance, a chosen location nearby Marseille would have a mean coverage over the year of about $32 \%$, which stands for a link availability of $68 \%$. This value might be good for a single station, but going through the quarters of the year, varying availabilities of $63 \%, 75 \%, 83 \%$ and $54 \%$ are observed.

\subsection{Ground-station diversity}

To raise link availability one can set up a system of several ground stations. Table II presents the availabilities in the quarterly periods of the year for four chosen locations. According to the 

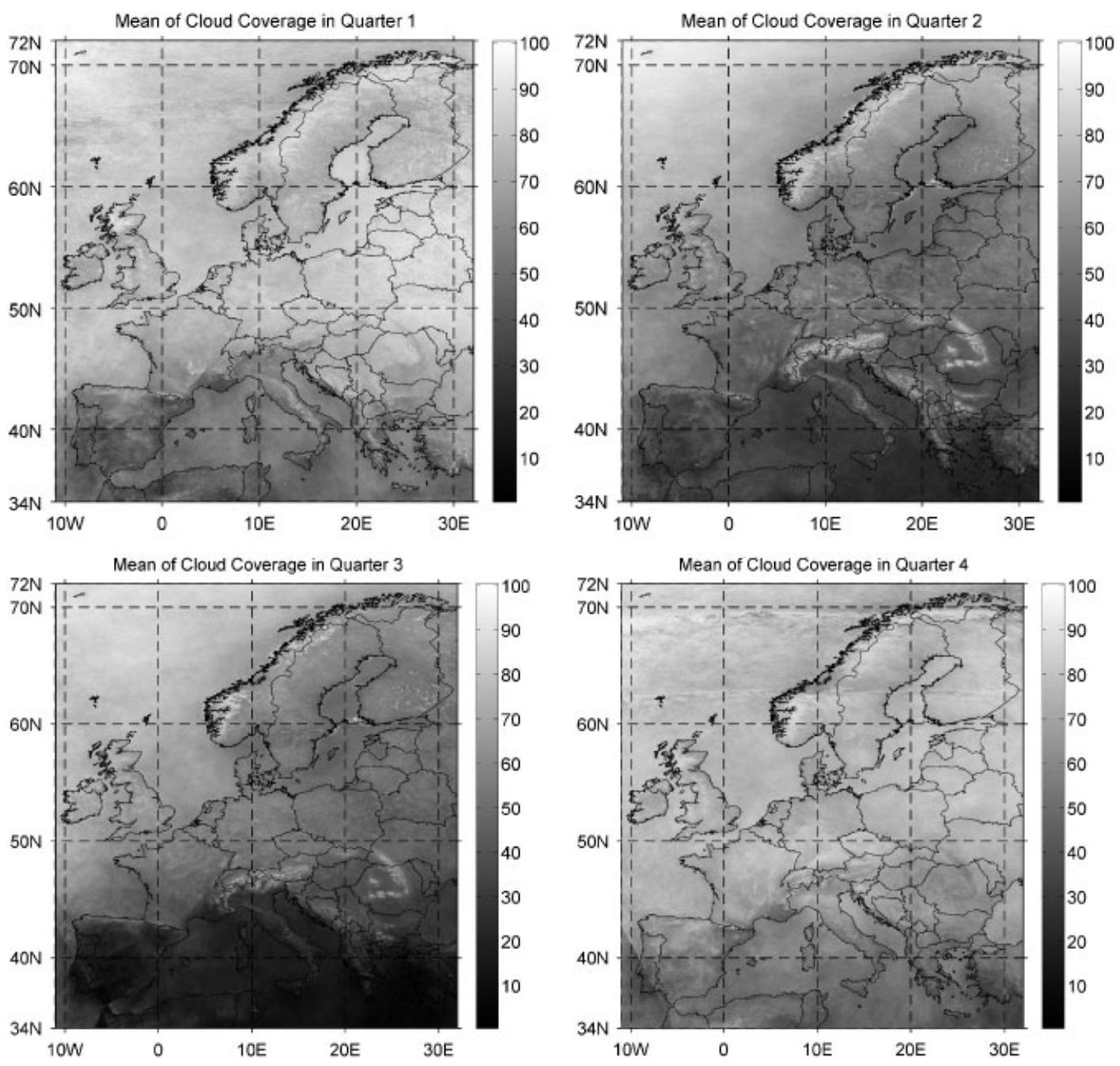

Figure 22. Mean of cloud cover over the quarterly periods of the year.

analysis of weather correlation in [28], the cloud cover correlation around the chosen locations for the years 2004/05 in dependence of the distance was determined. Figure 23 shows an exponential best-fit curve of the correlation coefficient values between Marseille and its surrounding locations. One can see the decrease in the correlation curve. Therefore, it is assumed that for distances over $900 \mathrm{~km}$ the weather cells are uncorrelated.

Hence, four sites with this least distance have been chosen to study compound link availability: the observatory on Calar Alto (Spain) and locations in the near of Marseille (France), Catania (Italy) and Heraklion (Greece) as illustrated in Figure 24. These sites feature low mean cloud coverage over the year and infrastructural accessibility. 
Table II. Individual link availabilities in percentage for the considered locations.

\begin{tabular}{lcccc}
\hline Station availability in & Heraklion (HER) & Catania (CAT) & Marseille (MAR) & Calar Alto (CAL) \\
\hline Quarter 1 & 57 & 62 & 63 & 61 \\
Quarter 2 & 83 & 72 & 75 & 62 \\
Quarter 3 & 97 & 87 & 81 & 78 \\
Quarter 4 & 63 & 54 & 54 & 49 \\
Year & 75.0 & 68.8 & 68.3 & 62.5 \\
\hline
\end{tabular}

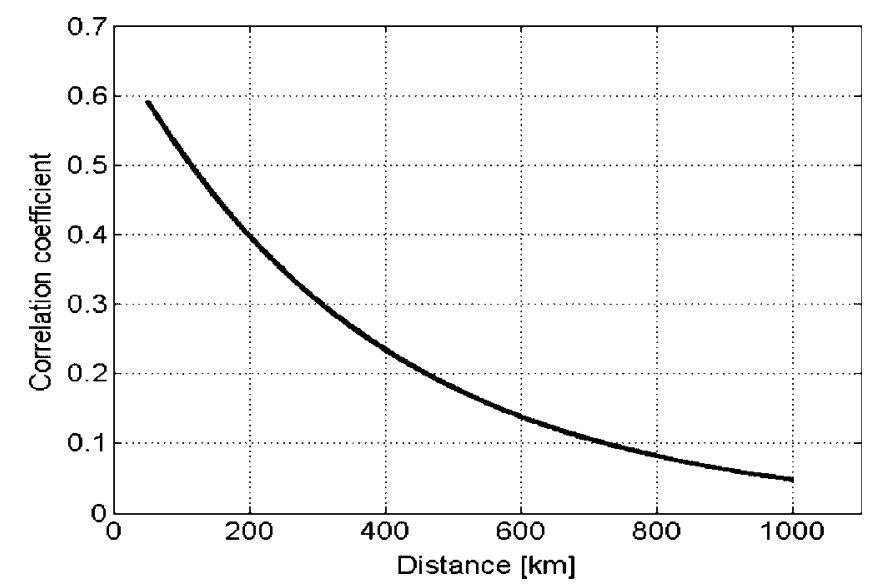

Figure 23. Best-fit curve for correlation over distance around Marseille.

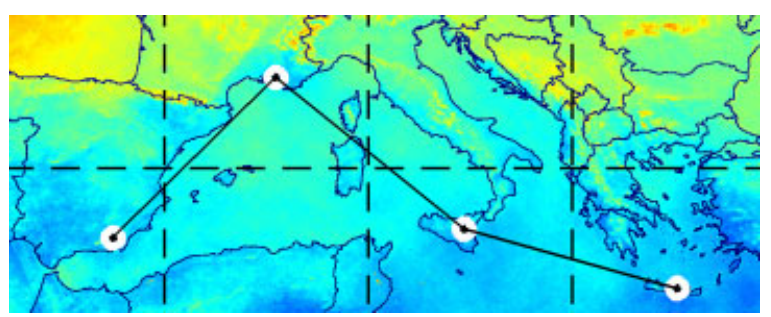

Figure 24. Locations of the stations. From left to right: Calar Alto Observatory, Marseille, Catania, Heraklion.

Table III gives the compound availabilities for different ground-station diversity systems. For this calculation, statistical independence of the weather conditions is assumed and the availability $p_{\text {div }}$ is given by

$$
p_{\text {div }}=1-\prod_{i=1}^{n} p_{i}
$$

with $p_{i}$ the probability of cloud cover of the $i$ th station and $n$ the number of stations used. The first system is set up with two stations having the best individual availabilities, the second with 
Table III. Joint availability for the different possibilities of ground station diversity.

\begin{tabular}{lccc}
\hline $\begin{array}{l}\text { System } \\
\text { availability in }\end{array}$ & $\begin{array}{c}\text { System 1 HER, } \\
\text { CAT }\end{array}$ & $\begin{array}{c}\text { System 2 HER, } \\
\text { CAT, MAR }\end{array}$ & $\begin{array}{c}\text { System 3 HER, } \\
\text { CAT, MAR, CAL }\end{array}$ \\
\hline Quarter 1 & 83.7 & 94.0 & 97.6 \\
Quarter 2 & 95.2 & 98.8 & 99.5 \\
Quarter 3 & 99.6 & 99.9 & 99.9 \\
Quarter 4 & 83.0 & 92.2 & 96.0 \\
Year & 90.4 & 96.2 & 98.3 \\
\hline
\end{tabular}

the three best and the third one with the four best. The link availability in system 3 is higher than $96 \%$ for each quarter and over $98 \%$ over the whole year. Especially remarkable is Quarter 3 which reaches an availability of $99.9 \%$ in systems 2 and 3. Reason for this is the extraordinary low cloud coverage over Heraklion for this time period. Remarkably, all quarters show an availability of over $90 \%$ for a joint of three stations and $95 \%$ for four stations. The system performance of the four stations joint could be further improved by searching for locations with a lower variance of cloud occurrence and still lower mean cloud coverage. This could result in a joint availability of $99.9 \%$ for the whole year.

\subsection{Wavelength dependency of cloud attenuation}

Avoiding clouds should be the first priority in stratospheric up/downlinks. But if this is not possible the influence of the cloud on the light beam attenuation has to be examined. To estimate the possible cloud attenuation, a calculation of the Mie scattering for the particle size distribution of a Stratus and a Cumulus cloud was done. A description of the formation and characteristics of theses cloud types is depicted in [29].

Stratus clouds are one of the most frequent clouds and normally reach vertical thicknesses between 200 and $800 \mathrm{~m} \mathrm{[30].} \mathrm{The} \mathrm{chosen} \mathrm{particle} \mathrm{size} \mathrm{distribution} \mathrm{for} \mathrm{this} \mathrm{cloud} \mathrm{is} \mathrm{based} \mathrm{on}$ LOWTRAN and FASCODE models [31]. For the Cumulus cloud, the $\mathrm{C} 1$ model from Dermendjian [32] was taken. The corresponding particle distribution functions can be seen in Figure 25 (left). Both calculations were performed using the Mie-tool from the libRadtran simulation suite [33]. Stratus has moderate attenuation and a vertical extension of only a few hundred meters.

In the right part of Figure 25, the total attenuation over wavelength for a given minimum and maximum geometrical thickness in vertical direction $z$ can be seen. This determines the minimum and maximum attenuation, respectively. The progression of the curves is qualitatively similar for the Cumulus and Stratus model. This can be observed with most of the cloud types having a similar particle size distribution. But there are cloud types with stronger (Nimbostratus, Cumulonimbus) or weaker (Altostratus, Stratocumulus) attenuation. Remarkable for most cloud types is the local minimum at wavelengths between 10 and $12 \mu \mathrm{m}$. A stratus cloud of $200-\mathrm{m}$ thickness features $22 \mathrm{~dB}$ less attenuation at $11.13 \mu \mathrm{m}$ compared with NIR wavelengths like $1.5 \mu \mathrm{m}$ which have been used for STROPEX. For future systems, $\mathrm{CO}_{2}$ Lasers or Quantum-Cascade Lasers could be used for wavelength which matches the attenuation minimum around $11.0 \mu \mathrm{m}$. However, for most clouds the attenuation even at this minimum is too high to establish an optical downlink. 

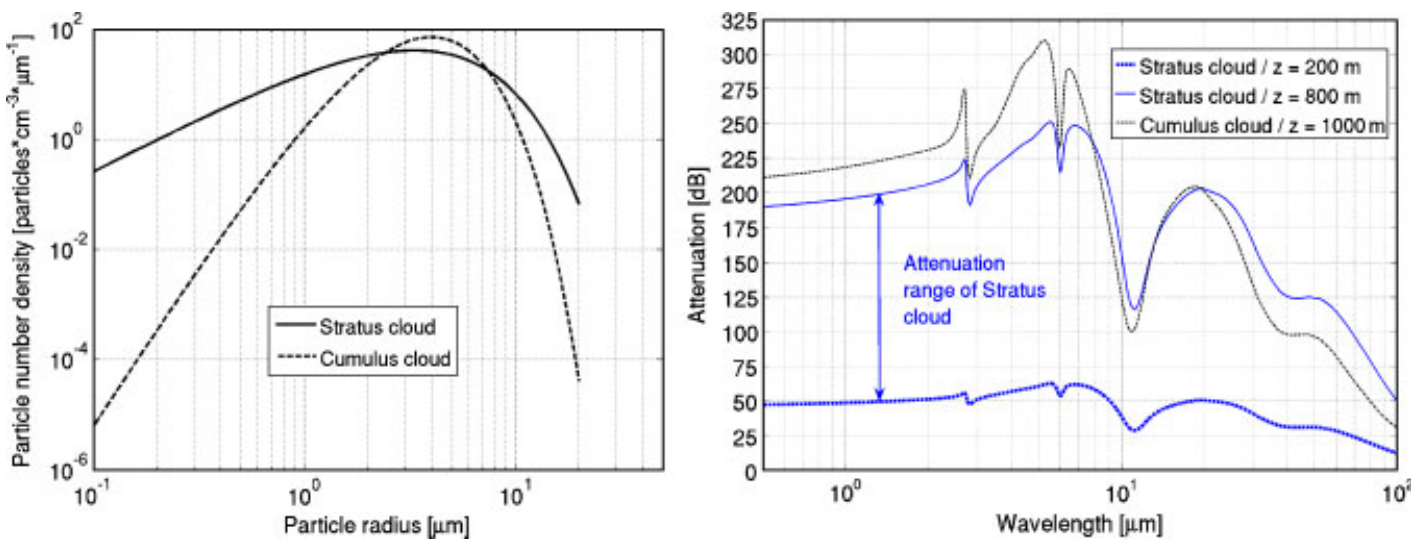

Figure 25. The size distribution of a Stratus cloud as it is used in LOWTRAN and FASCODE and a Cumulus C1 model of Dermendjian. The right graph depicts the attenuation variations of these models over the wavelengths.

\section{CONCLUSION}

The stratospheric optical experiment performed the first known optical Gbit/s downlink to a ground station. The chosen system concept with a relatively large transmit divergence of $1 \mathrm{mrad}$ for about $64 \mathrm{~km}$ resulted in a nearly error free transmission with $1.25 \mathrm{Gbit} / \mathrm{s}$ at $\mathrm{BER}<10^{-9}$ with a large pointing margin for the transmitter. The concept with an optical periscope also has the advantage that fast tip/tilt mirrors as fine pointing assembly can be easily incorporated into the design, allowing applications at stratospheric platforms which feature more complex vibration spectra at higher frequencies than the balloon in STROPEX. Blue sky background radiation is not a problem at $1550 \mathrm{~nm}$ because of the sufficiently low sun irradiance at this wavelength. BER did not change remarkably when the filter bandwidth in the receiver was changed from 10 to $60 \mathrm{~nm}$.

The measurement of the scintillation has shown that significant aperture averaging occurred during the trial, preventing deep fades from deteriorating the data reception. The intensity scintillation index $\sigma_{I}^{2}$ ranged from 0.28 to 1.12 , and the PDF of the intensity was lognormal only when the scintillation index had low values. Although the coherence length had no impact on the IM/DD system, it has been measured for investigations of possible other modulation schemes.

Considering pessimistic turbulence parameters, the link budget of the experiment has been reviewed and compared with that of a coherent transmission link. It was shown that the coherent link, which must feature a smaller receiving aperture, provides a lower link power margin.

A ground-station diversity system in southern Europe with a combination of four stations is able to assure a high availability over the whole year. For improvement of individual and joint link availability one can use a wavelength of around $11 \mu \mathrm{m}$ which is able to propagate through thin clouds. 


\section{APPENDIX: ABBREVIATIONS}

$\begin{array}{ll}\text { APD } & \text { Avalanche photo diode } \\ \text { BER } & \text { bit error rate } \\ \text { BSC } & \text { beam splitter cube } \\ \text { CCD } & \text { charge coupled device } \\ \text { DD } & \text { direct detection } \\ \text { DIMM } & \text { differential image motion monitor } \\ \text { ECC } & \text { European cloud climatology } \\ \text { HAP } & \text { high-altitude platform } \\ \text { FSO } & \text { free-space optics } \\ \text { FOV } & \text { field of view } \\ \text { FELT } & \text { free-space experimental laser terminal } \\ \text { IM/DD } & \text { intensity modulation with direct detection } \\ \text { NIR } & \text { near-infrared } \\ \text { NOAA } & \text { National Oceanic and Atmospheric Administration } \\ \text { PRBS } & \text { pseudo random binary sequence } \\ \text { PAT } & \text { pointing acquisition and tracking } \\ \text { STROPEX } & \text { stratospheric optical payload experiment } \\ \text { TOGS } & \text { transportable optical ground station } \\ \text { TMTC } & \text { telemetry and tele-command } \\ \text { Tx } & \text { transmitter } \\ \text { Rx } & \text { receiver } \\ \text { TP } & \text { turbulence profiler }\end{array}$

\section{ACKNOWLEDGEMENTS}

The CAPANINA/STROPEX project was supported by the European Commission, contract number FP6IST-2003-506745. The ECC Data are provided by the courtesy of Remote Sensing Data Center (DFD) in the German Aerospace Centre in Oberpfaffenhofen.

\section{REFERENCES}

1. CAPANINA Website. September 2006. http://www.capanina.org

2. Grace D, Capstick MH, Mohorcic M, Horwath J, Pallavicini MB, Fitch M. Integrating users into the wider broadband network via high altitude platforms. IEEE Wireless Communications 2005; 12:98-105.

3. Giggenbach D, Purvinskis R, Werner M, Holzbock M. Stratospheric optical inter-platform links for high altitude platforms. AIAA-Proceedings of the 20th ICSSC, May 2002.

4. Horwath J, Grace D, Giggenbach D, Knapek M, Perlot N. Optical communication from HAPs-overview of the stratospheric optical payload experiment (STROPEX). AIAA-Proceedings of the 22nd ICSSC, May 2004.

5. Barry JR, Lee E. Performance of coherent optical receivers. Proceedings of the IEEE 1990; 78(8):1369-1394.

6. Giggenbach D, Horwath J. Optical free-space communications downlinks from stratospheric platforms-overview on STROPEX, the optical communications experiment of CAPANINA. Proceedings of the 2005 IST Mobile Summit, 2005.

7. Knapek M, Horwath J, Perlot N, Wilkerson B. The DLR ground station in the optical payload experiment (STROPEX) - results of the atmospheric measurement instruments. Proceedings of the SPIE 2006; 6304.

8. Tatarskii VI. The Effects of the Turbulent Atmosphere on Wave Propagation. National Technical Information Service, Springfield, 1971. 
9. Tokovinin A, Kornilov V. Measuring turbulence profile from scintillations of single stars. Astronomical Site Evaluation in the visible and Radio Range, Benkhaldoun Z, Muñoz-Tuñon C, Vernin J (eds). ASP Conference Series, vol. 266. 2002; 104-112.

10. Tokovinin A, Kornilov V, Shatsky N, Voziakova O. Restoration of turbulence profile from scintillation indices. Monthly Notices of the Royal Astronomical Society 2003; 343(3):891-899.

11. Caccia JL, Azouit M, Vernin J. Wind and $C_{n}^{2}$ profiling by single-star scintillation analysis. Applied Optics 1987; 26(7):1288-1294.

12. Andrew LC, Phillips RL. Laser Beam Propagation through Random Media (2nd edn). SPIE Optical Engineering Press: Bellingham, WA, 1998.

13. Sarazin M, Roddier F. The ESO differential image motion monitor. Astronomy and Astrophysics 1990; 227: 294-300.

14. Vernin J, Munoz-Tunon C. Measuring astronomical seeing: the DA/IAC DIMM. Publications of the Astronomical Society of the Pacific 1995; 107(709):265-272.

15. Vernin J, Munoz-Tunon C. Optical seeing at La Palma Observatory. 2: Intensive site testing campaign at the Nordic Optical Telescope. Astronomy and Astrophysics 1994; 284:311-318.

16. Andrews LC, Phillips RL, Hopen CY. Laser Beam Scintillation with Applications. SPIE Optical Engineering Press: Bellingham, WA, 2001.

17. Gagliardi R, Karp S. Optical Communications. Wiley: New York, 1995.

18. Henniger H, Giggenbach D, Horwath J, Rapp C. Evaluation of optical up- and downlinks from high altitude platforms using IM/DD. Proceedings of the SPIE 2005; 5712:24-36.

19. Kiasaleh K. On the probability density function of signal intensity in free-space optical communications systems impaired by pointing jitter and turbulence. Optical Engineering 1994; 33(11):3748-3757.

20. Perlot N. Evaluation of the scintillation loss for optical communication systems with direct detection. Optical Engineering 2007; 46(2).

21. Fried DL. Optical heterodyne detection of an atmospherically distorted signal wave front. Proceedings of the IEEE 1967; 55:57-66.

22. Cohen SC. Heterodyne detection: phase front alignment, beam spot size, and detector uniformity. Applied Optics 1975; 14:1953-1959.

23. Harding CM, Johnston RA, Lane RG. Fast simulation of a Kolmogorov phase screen. Applied Optics 1999; 38:2161-2170.

24. Winick KA. Atmospheric turbulence-induced signal fades on optical heterodyne communication links. Applied Optics 1986; 25:1817-1825.

25. Toyoshima $\mathrm{M}$ et al. Long-term statistics of laser beam propagation in an optical ground-to-geostationary satellite communications link. IEEE Transactions on Antennas and Propagation 2005; 53(2):842-850.

26. Lange R, Smutny B, Wandernoth B, Czichy R, Giggenbach D. 142 km, 5.625 Gbps free-space optical link based on homodyne BPSK modulation. Proceedings of the SPIE 2006; 6105:61050A 1-61050A 9.

27. Wandernoth B. 20 photon/bit $565 \mathrm{Mbit} / \mathrm{s}$ PSK homodyne receiver using synchronisation bits. IEEE Electronics Letters 1992; 28:387-388.

28. Garcia P, Benarroch A, Riera J. Spatial distribution of cloud cover: effect on multi-beam satellite links. Proceedings of the International Workshop of COST Actions 272 and 280, 2003; 205-212.

29. Kyle TG. Atmospheric Transmission. Pergamon Press: Oxford, 1991.

30. Churnside JH, Shaik K. Atmospheric propagation issues relevant to optical communications. NOAA Technical Memorandum ERL WPL-159, Boulder, CO, 1989.

31. Shettle EP. Models of aerosols, clouds and precipitation for atmospheric propagation studies. Proceedings of the AGARD 1998; 454:15/1-13.

32. Dermendjian D. Electromagnetic Scattering on Spherical Polydispersions. American Elsevier: New York, 1969.

33. Kylling A, Mayer B. LibRadtran: Library for Radiative Transfer Calculations, Edition 1.0 for LibRadtran Version $1.1-$ beta, 2005. 


\section{AUTHORS’ BIOGRAPHIES}

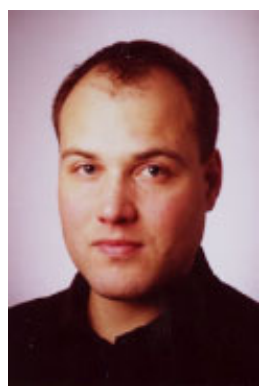

Joachim Horwath has been a member of the German Aerospace Centre, Institute of Communications and Navigation since 2002. His work area is optical free space communication and atmospheric effects on optical rays. He managed several DLR activities of projects that dealt with communication for High Altitude Platforms and was the project leader of the CAPANINA STRatospheric Optical Payload Experiment (STROPEX). He is involved in the Atmospheric Transmission Monitor Project with the coherent optical downlink from the TerraSAR-X Satellite and responsible for optical communication payload design of an airborne optical communication terminal for remote sensing missions.

Nicolas Perlot received his MS and his $\mathrm{PhD}$ in electronics engineering at the University of Valenciennes (France) in 2002 and 2005, respectively. He joined the Optical Communication Group of the German Aerospace Center (DLR) in Oberpfaffenhofen (Germany) in 2002. His research interests are: atmospheric beam propagation, free-space optical communications, characterization and simulation of random processes.

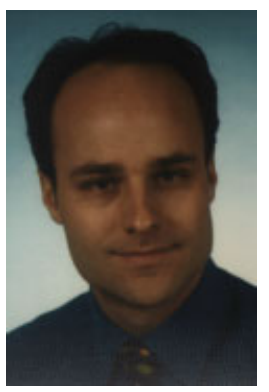

Markus Knapek has been a member of the German Aerospace Centre, Institute of Communications and Navigation since 2003. He has been responsible for the design and construction of the optical ground station and the development of the atmospheric measurement instruments. In 2007 he supervises the atmospheric measurements in the optical downlink experiments from the coherent Laser Communication Terminal LCT on TerraSAR-X. Since 2005 he is leader of the optical workgroup in COST297 HAPCOS.

Florian Moll has been a member of the German Aerospace Centre, Institute of Communications and Navigation since 2006. As a student of the University of Applied Sciences Jena he made his diploma thesis at the DLR about atmospheric attenuation and cloud coverage statistics. After graduating he kept on working at the DLR involved in several research areas and started a Master course at the Technical University Munich (TUM). 